\title{
LONG-RANGE POTENTIAL SCATTERING BY ENSS'S METHOD IN TWO HILBERT SPACES ${ }^{1}$
}

\author{
DENIS A. W. WHITE
}

\begin{abstract}
Existence and completeness of wave operators is established by a straightforward transposition of the original short range result of Enss into an appropriate two-Hilbert space setting. Applied to long range quantum mechanical potential scattering, this result in conjunction with recent work of Isozaki and Kitada reduces the problem of proving existence and completeness of wave operators to that of approximating solutions of certain partial differential equations on cones in phase space. As an application existence and completeness of wave operators is established for Schrödinger operators with a long range multiplicative and possibly rapidly oscillating potential.
\end{abstract}

1. Introduction. The geometric time-dependent methods of Enss now play a fundamental role in the study of quantum mechanical potential scattering theory. However, until recently the success of this method has been most dramatic in the treatment of short range interactions. All this has been changed by the recent work of Isozaki and Kitada [20]. Their approach, using "time-independent modifiers" allows them to apply Enss's arguments to prove existence and completeness of wave operators for Schrödinger operators with long range potentials.

In the present paper we transpose the usual short range result of Enss into an appropriate two-Hilbert space setting. Applied to the study of long range scattering, in conjunction with (our version of) Isozaki and Kitada's "modifiers", this result reduces the problem of proving existence and completeness of wave operators to that of approximating solutions of certain partial differential equations on cones in phase space. In the context of Isozaki and Kitada's result [20] this equation is an eikonal equation and we use their solution to give an alternate proof of their result. Then we extend this result to allow more general potentials.

The following elementary argument will elucidate the problems to be encountered below as well as the relationship between this work and [20]. Let $H_{0}$ and $H$ be selfadjoint operators on $L^{2}\left(\mathbf{R}^{n}\right), H_{0}=-\frac{1}{2} \Delta$ and $H$ is a long range perturbation of $H_{0}: H=H_{0}+V$. The usual approach to scattering theory for $H$ and $H_{0}$ is to introduce the (Dollard) modified wave operators

$$
\Omega_{m}^{ \pm}=\underset{t \rightarrow \mp \infty}{s-\lim } e^{i t H} e^{-i W(t, \nabla / i)}
$$

for a suitably chosen function $W: \mathbf{R} \times \mathbf{R} \rightarrow \mathbf{R}$. ("s-lim" indicates the limit in the strong operator topology.) In contrast Isozaki and Kitada [20] introduce the "two-space" wave operators (or "time-independent modifiers")

$$
\Omega^{ \pm}(J)=\underset{t \rightarrow \mp \infty}{\mathrm{s}-\lim ^{i t H}} J e^{-i t H_{0}},
$$

Received by the editors December 7, 1984.

1980 Mathematics Subject Classification. Primary 35P25, 81F05; Secondary 47A40.

${ }^{1}$ Research supported by the Natural Science and Engineering Research Council of Canada. 
where $J$ is a bounded operator. Which are more general, $\Omega_{m}^{ \pm}$or $\Omega^{ \pm}(J)$ ? If $\Omega_{m}^{ \pm}$ exist, then $\Omega_{m}^{ \pm} e^{-i t H_{0}}=e^{-i t H} \Omega_{m}^{ \pm}$provided $W$ is "reasonable"; see $[\mathbf{1 5}$, p. 75]. Therefore if we define $J=\Omega_{m}^{-}$, then

$$
e^{i t H} J e^{i t H_{0}}=e^{i t H} e^{-i t H} J=J=\Omega_{m}^{-} .
$$

Thus if $\Omega_{m}^{-}$exists it is possible, at least in theory, to choose $J$ so that $\Omega^{-}(J)$ exists and is $\Omega_{m}^{-}$. This argument also suggests a plausible choice of $J$ even when $\Omega_{m}^{-}$are not known but expected to exist: $J$ should approximate $\Omega_{m}^{-}$. However, a moment's reflection about the physical interpretation of $\Omega_{m}^{-}$will convince the reader that $\Omega_{m}^{-}$ would be very difficult to approximate in general. At this stage the idea of V. Enss to introduce incoming and outgoing states can rescue us. Although $\Omega_{m}^{-} \phi$ might be difficult to approximate for general states $\phi$, it should be possible to approximate $\Omega_{m}^{-} \phi_{\text {out }}$ if $\phi_{\text {out }}$ is an outgoing state. Conceivably $J$ could be constructed so that $\Omega_{m}^{-}-J$ is small (i.e. compact) on the outgoing states. Actually, since $\Omega_{m}^{-}$is unknown the appropriate goal is to choose $J$ so that $H J-J H_{0}$ restricted to outgoing states is short range. (Recall Cook's method.) There is nothing special about $\Omega_{m}^{-}$. Interchanging $\Omega_{m}^{-}$with $\Omega_{m}^{+}$and outgoing with incoming in the above argument, we should be able to choose a (very different) operator $J$ which we denote $J^{+}$which approximates $\Omega_{m}^{+}$. The $J$ which approximates $\Omega_{m}^{-}$is denoted $J^{-}$. The actual construction of $J^{ \pm}$is due to Isozaki and Kitada. We show that $H^{-}-J^{-} H_{0}$ (resp. $\mathrm{HJ}^{+}-J^{+} \mathrm{H}_{0}$ ) restricted to outgoing (resp. incoming) states is short range and provides an extension of the Enss results (Theorem 3.1 and Corollary 4.4) which prove the existence and completeness of $\Omega^{ \pm}\left(J^{ \pm}\right)$.

In contrast Isozaki and Kitada piece together $J^{+}$and $J^{-}$into an operator $J$ and then prove the existence and completeness of $\Omega^{ \pm}(J)$. (Their $\Omega^{-}(J)$ (resp. $\Omega^{+}(J)$ ) equals our $\Omega^{-}\left(J^{-}\right)$(resp. $\Omega^{+}\left(J^{+}\right)$).) However $H J-J H_{0}$ is not short range whereas $\mathrm{HJ}^{-}-\mathrm{J}^{-} \mathrm{H}_{0}$ and $\mathrm{HJ}^{+}-\mathrm{J}^{+} \mathrm{H}_{0}$ are short range on complementary subspaces.

The existence of $\Omega^{ \pm}\left(J^{ \pm}\right)$immediately implies that of $\Omega^{ \pm}(J)$, where $J$ is as in $[20]$ or $J=J^{-} E_{D}([0, \infty))+J^{+} E_{D}((-\infty, 0])$. Here $E_{D}$ is the spectral measure of $D=\frac{1}{2}[(\nabla / i) \cdot x+x \cdot(\nabla / i)]$ (see $\left.[\mathbf{1 1},(9.29)]\right)$. Moreover $\Omega^{-}\left(J^{-}\right)=\Omega^{-}(J)$ and $\Omega^{+}\left(J^{+}\right)=\Omega^{+}(J)$ so that nothing is lost by working with $\Omega^{ \pm}\left(J^{ \pm}\right)$.

The reader may have noticed a disturbing consequence of (1.2) which deserves mention. If $J=\Omega_{m}^{-}$, then $\Omega^{ \pm}(J)$ both exist and both are $\Omega_{m}^{-}$!. Thus $\Omega^{ \pm}(J)$ cannot in general be expected to have the same physical significance as $\Omega_{m}^{ \pm}$. (For the significance of $\Omega_{m}^{ \pm}$, see [38 or 18], for example.) This difficulty is circumvented here because $\Omega^{ \pm}\left(J^{ \pm}\right)=\Omega_{m}^{ \pm}$by $(4.2)$ of $[20]$.

1(a). Results. We shall apply our abstract results Theorem 3.1 and Corollary 4.4 to prove the existence and completeness of the wave operators (1.1) when $H_{0}$ and $H$ are the selfadjoint operators on $L^{2}\left(\mathbf{R}^{n}\right)$,

$$
\begin{aligned}
& \left.H_{0}=-\frac{1}{2} \Delta \quad \text { (with domain the Sobolev space } \mathbf{H}^{2}\left(\mathbf{R}^{n}\right)\right) \\
& H=H_{0}+\frac{1}{2}(\Delta U)+\frac{1}{2}|\nabla U|^{2}+V+V_{s}
\end{aligned}
$$

We assume

(LR1) $V \in C^{\infty}\left(\mathbf{R}^{n}\right)$ is real valued and for some $\varepsilon_{0}>0$

$$
\left|D^{\alpha} V(x)\right|<C_{\alpha}\langle x\rangle^{-|\alpha|-\varepsilon_{0}} \quad \text { for all } \alpha \in \mathbf{N}^{n}
$$

where $\langle x\rangle=\left(1+|x|^{2}\right)^{1 / 2}$; 
(LR2) $U \in L^{\infty}\left(\mathbf{R}^{n}\right)$ is real valued and $h$ defined by $h(r)=\|F(|x|>r) U\|$ belongs to $L^{1}((0, \infty))$, where $F(|x|>r)$ is the operator of multiplication by the indicator function for the set $\{x:|x|>r\}$;

and for some $\gamma>0$

(SR) $V_{s}$ is a symmetric operator, $V_{s}\left(H_{0}+1\right)^{-\gamma}$ is compact and for some $k \in \mathbf{N}$, $h$ defined by $h(r)=\left\|\left(H_{0}+1\right)^{-k} g_{1}(x / r) V_{s}\left(H_{0}+1\right)^{-\gamma}\right\|$ belongs to $L^{1}((0, \infty))$, where $g_{1} \in C^{\infty}\left(\mathbf{R}^{n}\right), 0 \leq g_{1} \leq 1$, and

$$
g_{1}(x)= \begin{cases}1 & \text { if }|x|>2 \\ 0 & \text { if }|x|<1\end{cases}
$$

Assumption (LR1) can be weakened (see [15, Proposition 3.5]). The assumption (LR2) does not include smoothness conditions for $U$, so that $H$ may not make sense as written above. Following Combescure [3] we write

$$
H=\frac{1}{2} e^{-U}\left(\frac{1}{i} \nabla\right) e^{2 U}\left(\frac{1}{i} \nabla\right) e^{-U}+V+V_{s},
$$

where $e^{-U}$ acts multiplicatively. Then $H$ is selfadjoint; see (7.4) below.

The operators $J^{ \pm}$introduced above will be defined as

$$
J^{ \pm} \phi(x)=(2 \pi)^{-n / 2} e^{U(x)} \int_{n} e^{i(x \cdot \xi)+i \theta^{ \pm}(x, \xi)} \hat{\phi}(\xi) d \xi,
$$

where $\hat{\phi}$ is the Fourier transform of $\phi$ and $\theta^{ \pm}$are solutions of the eikonal equations: for $|x|,|\xi|$ large enough

$$
\frac{1}{2}\left|\xi+\nabla_{x} \theta^{ \pm}(x, \xi)\right|^{2}+V(x)=\frac{1}{2}|\xi|^{2} \quad \text { if } \mp(x \cdot \xi)>-\frac{1}{2}|x||\xi| .
$$

We describe $\theta^{ \pm}$more carefully in $\S 5$. The significance of the eikonal equation can best be appreciated by formally computing $H J^{ \pm}-J^{ \pm} H_{0}$ as in (7.4) below.

Recall that the subspace of continuity of a selfadjoint operator is the orthogonal complement of the space spanned by its eigenvectors.

THEOREM 1.1. Suppose $V$ and $U$ satisfy (LR1) and (LR2) respectively and $V_{s} e^{U}$ satisfies $(\mathrm{SR})_{1 / 2}$ (replace $V_{s}$ there by $\left.V_{s} e^{U}\right)$ or if $U=0, V_{s}$ satisfies (SR) $)_{1}$. Let $H_{0}, H$ and $J^{ \pm}$be defined by (1.3), (1.5) and (1.6). Then $H$ is selfadjoint with essential spectrum $[0, \infty)$ and the wave operators $\Omega^{ \pm}\left(J^{ \pm}\right)$of $(1.1)$ exist, are isometries and have ranges both equal to the subspace of continuity of $H$. In particular $\Omega^{ \pm}\left(J^{ \pm}\right)$are asymptotically complete and $H$ has no singularly continuous spectrum.

An additional conclusion about $\Omega^{ \pm}\left(J^{ \pm}\right)-J^{ \pm}$can be drawn (see Remark 7.1).

This result seems to be the first to combine the two types of long range behaviour, the slow decay of (LR1) and the oscillatory potentials allowed by (LR2). Scattering theory has been discussed extensively for potentials satisfying (LR1). For example see $[1,6,10,11,16-20,22,23,25,26,27,30,31,33,37,38,45,46]$. Our approach to oscillatory potentials follows Combescure $[\mathbf{3}]$ and Pearson $[\mathbf{3 4}]$ and does not include all the moderately oscillating potentials of Dollard and Friedman [8] (see also $[\mathbf{4}, \mathbf{5}]$ ). The reader interested in Enss's geometric approach to scattering theory should see $[3,9-14,32,35,36,37,42,44]$. The two-Hilbert space wave operators (1.1) have also been employed in a similar context in $[18,20-23,39]$. For more complete references see the "Notes" of [42] (for before 1978), [11] (Enss approach) and [47] (oscillatory potentials) or [36]. 
EXAMPLE 1.2. Theorem 1.1 is applicable to $H$ defined formally on $L^{2}\left(\mathbf{R}^{n}\right)$, $n \geq 2$, as

$$
H=H_{0}+a r^{-\beta} \sin b r^{\alpha} g_{1}(x)+a^{\prime} r^{-\beta^{\prime}} \sin b^{\prime} r^{\alpha^{\prime}}\left(1-g_{1}(x)\right)+V+V_{s}
$$

$(r=|x|)$, where $\beta+\alpha>1$ and $\beta+2 \alpha>3 ; \beta^{\prime}+\alpha^{\prime}<1$ and $\alpha^{\prime}<0$ and $a, b, a^{\prime}, b^{\prime} \in \mathbf{R}$, $g_{1} \in C^{\infty}\left(\mathbf{R}^{n}\right)$ satisfies (1.4) and $V$ satisfies (LR1) and $V_{s}$ acts multiplicatively and satisfies $(\mathrm{SR})_{1 / 2}$.

We shall briefly discuss Example 1.2 in $\S 7$.

1(b). Contents. We introduce the incoming and outgoing spaces in $\S 2$ but relegate the proofs to the Appendix. $\S \S 3$ and 4 contain the abstract existence and completeness results. We define the operators $J^{ \pm}$in $\S 5$ and derive their basic properties and in $\S 6$ apply them to prove Theorem 6.1 which is just Theorem 1.1 when $U=0$. In $\S 7$ we prove Theorem 1.1 and discuss Example 1.2.

$1(\mathrm{c})$. Notation. We use the following notation (for other notation see the beginning of $\S 3)$ :

$\mathbf{N}=\{0,1,2, \ldots\}$

$\mathbf{R}$ and $\mathbf{C}$ are the real and complex numbers;

$|\alpha|=\alpha_{1}+\cdots+\alpha_{n}$ if $\alpha=\left(\alpha_{1}, \ldots, \alpha_{n}\right)$ in $\mathbf{N}^{n}$;

$D_{x}^{\alpha}=(1 / i)^{|\alpha|} \partial^{\alpha_{1}} / \partial x_{1}^{\alpha_{1}} \cdots \partial^{\alpha_{n}} / \partial x_{n}^{\alpha_{n}}(i=\sqrt{-1})$;

$\Delta=\partial^{2} / \partial x_{1}^{2}+\cdots+\partial^{2} / \partial x_{n}^{2}$

$\partial_{x} f(x, y)$-(Fréchet) derivative of $f(\cdot, y)$ ( $y$ fixed);

$C^{\infty}\left(\mathbf{R}^{n}\right)$-infinitely differentiable complex valued functions on $\mathbf{R}^{n}$;

$S\left(\mathbf{R}^{n}\right) \subseteq C^{\infty}\left(\mathbf{R}^{n}\right)$-Schwartz space;

$C_{0}^{\infty}(U)$-all $\phi \in C^{\infty}\left(\mathbf{R}^{n}\right)$ with support compact and in $U$;

$F(M)$-operator of multiplication by the indicator function for the Borel set $M$;

$d_{1} x=(2 \pi)^{-n / 2} d x$ (i.e. $(2 \pi)^{-n / 2}$ times Lebesgue measure on $\mathbf{R}^{n}$ );

$\mathcal{F} \phi(\xi)=\phi(\xi)=\int_{\mathbf{R}^{n}} e^{i x \cdot \xi} \phi(x) d_{1} x$ (Fourier transform);

$(\phi \mid \psi)=\int_{\mathbf{R}^{n}} \phi(x) \overline{\psi(x)} d x$ (inner product on $L^{2}\left(\mathbf{R}^{n}\right)$ );

$\|\phi\|=(\phi \mid \phi)^{1 / 2}$

$C_{\lambda}$ is a constant depending on a parameter $\lambda$;

det is the determinant (of a matrix);

$\langle x\rangle=\left(1+|x|^{2}\right)^{1 / 2}$.

2. The incoming and outgoing subspaces. We introduce at this early stage the incoming and outgoing subspaces because, for the reasons mentioned in the Introduction, they will be required to even state our hypotheses. For the definition of these subspaces, we follow Davies [48] primarily for unity of exposition; all our operators will be integral operators. Other definitions would work just as well provided Theorem 2.2, and Lemmas 6.3 and 6.4 below remain valid.

For each $\sigma,-1 \leq \sigma \leq 1$, define

$$
M_{\sigma}^{ \pm}=\left\{(y, p) \in \mathbf{R}^{2 n}: \mp y \cdot p>\sigma|y||p|\right\}
$$

("dot" denotes the usual product). Intuitively $\phi \in L^{2}\left(\mathbf{R}^{n}\right)$ is outgoing (resp. incoming) if $\phi(y)$ and its Fourier transform $\hat{\phi}(p)$ are nonzero only when $(y, p) \in M_{\sigma}^{-}$ (resp. $(y, p) \in M_{\sigma}^{+}$). Of course we cannot expect such a naive approach to work because of the uncertainty principle and so we proceed as follows. Choose $\eta$ in $S\left(\mathbf{R}^{n}\right)$ (the Schwartz space) so that $\eta$ and $\hat{\eta}$ are real valued and

$$
\|\eta\|=(2 \pi)^{-n / 2}, \quad \hat{\eta} \in C_{0}^{\infty}\left(\mathbf{R}^{n}\right) \quad \text { and } \operatorname{supp} \hat{\eta}=\{\xi:|\xi|<\delta\}
$$


where $0<\delta<1$ is a parameter to be specified ( $\operatorname{supp} \hat{\eta}$ denotes the support of $\hat{\eta}$ ). For each $(y, p) \in \mathbf{R}^{n} \times \mathbf{R}^{n}$ define

$$
\eta_{y, p}(x)=e^{i p \cdot(x-y)} \eta(x-y)
$$

so that

$$
\hat{\eta}_{y, p}(\xi)=e^{-i \xi \cdot y} \hat{\eta}(\xi-p) .
$$

Further define the linear mapping $\tau_{n}$ of $L^{2}\left(\mathbf{R}^{n}\right)$ into $L^{2}\left(\mathbf{R}^{2 n}\right)$ :

$$
\left(\tau_{\eta} \phi\right)(y, p)=\left(\phi \mid \eta_{y, p}\right)=\left(\hat{\phi} \mid \hat{\eta}_{y, p}\right)
$$

Then $\tau_{\eta} \phi(y, p)$ is a weighted average of the Fourier transform of the translate $\phi(\cdot+y)$, over a ball of radius $\delta$ centered at $p$.

LEMMA 2.1. The mapping $\tau_{\eta}$ is an isometry of $L^{2}\left(\mathbf{R}^{n}\right)$ into $L^{2}\left(\mathbf{R}^{2 n}\right)$. Moreover $\tau_{\eta}\left(S\left(\mathbf{R}^{n}\right)\right) \subseteq S\left(\mathbf{R}^{2 n}\right)$.

PROOF. For $\phi \in S\left(\mathbf{R}^{n}\right)$ we have

$$
\begin{aligned}
\left\|\tau_{\eta} \phi\right\|^{2} & =\int_{\mathbf{R}^{n}} \int_{\mathbf{R}^{n}}\left|\int_{\mathbf{R}^{n}} e^{-i p \cdot(x-y)} \eta(x-y) \phi(x) d x\right|^{2} d p d y \\
& =(2 \pi)^{n} \int_{\mathbf{R}^{n}} \int_{\mathbf{R}^{n}}|\eta(x-y)|^{2}|\phi(x)|^{2} d x d y=\|\phi\|^{2}
\end{aligned}
$$

by Plancherel's theorem. Therefore $\tau_{\eta}$ is an isometry. The verification that $\tau_{\eta} \phi \in$ $S\left(\mathbf{R}^{2 n}\right)$ is straightforward.

For each $f$ in $C_{0}^{\infty}((0, \infty))$ and each $\sigma,-1 \leq \sigma \leq 1$, define the outgoing operator $R_{\sigma}^{-}(f)$ and incoming operator $R_{\sigma}^{+}(f)$ as

$$
R_{\sigma}^{ \pm}(f)=\bar{f}\left(H_{0}\right) \tau_{\eta}^{*} F\left(M_{\sigma}^{ \pm}\right) \tau_{\eta} f\left(H_{0}\right)
$$

Here $F(M)$ denotes the operator of multiplication by the indicator function for the Borel set $M$. Also $\bar{f}$ is the complex conjugate of $f$ and $H_{0}=-\frac{1}{2} \Delta$. Clearly $R_{\sigma}^{ \pm}(f)$ are bounded Hermitian operators and

$$
R_{\sigma}^{-}(f)+R_{-\sigma}^{+}(f)=|f|^{2}\left(H_{0}\right)
$$

and

$$
\overline{R_{\sigma}^{-}(f) \phi}=R_{\sigma}^{+}(\bar{f}) \bar{\phi}
$$

For later convenience we record the identity

$$
\left.\left(\tau_{\eta}^{*} \Psi\right) \gamma \xi\right)=\int_{\mathbf{R}^{n}} \int_{\mathbf{R}^{n}} e^{-i \xi \cdot y} \hat{\eta}(\xi-p) \Psi(y, p) d y d p \quad \text { if } \Psi \in L^{1}\left(\mathbf{R}^{2 n}\right) \cap L^{2}\left(\mathbf{R}^{2 n}\right)
$$

which is easily verified by applying Fubini's theorem to $\left.\left(\Psi \mid \tau_{\eta} \phi\right)\left(=\left(\left(\tau_{\eta}^{*} \Psi\right)\right\rceil \hat{\phi}\right)\right)$.

The operators $R_{\sigma}^{ \pm}(f)$ depend on the choice of the parameter $\delta$ in (2.2) although this is not indicated in the notation. We shall assume that $\delta$ is so small that the following result is applicable. 
THEOREM 2.2. If $f, f_{1} \in C_{0}^{\infty}((0, \infty))$ and $-1<\sigma_{1}<\sigma<1$, then $R_{\sigma}^{ \pm}(f) \phi \in$ $S\left(\mathbf{R}^{n}\right)$ whenever $\phi \in S\left(\mathbf{R}^{n}\right)$ and

(a) for $\delta>0$ sufficiently small $\left(\delta=\delta\left(\sigma-\sigma_{1}, f, f_{1}\right)\right), R_{-\sigma_{1}}^{ \pm}\left(f_{1}\right) e^{-i t H_{0}} R_{\sigma}^{\mp}(f)$ is compact for all $\pm t>0$, and for every $l \in \mathbf{N}$

$$
\left\|R_{-\sigma_{1}}^{ \pm}\left(f_{1}\right) e^{-i t H_{0}} R_{\sigma}^{\mp}(f)\right\|<C_{l}(1+|t|)^{-l} \quad \text { if } \pm t>0
$$

(b) for $\delta>0$ sufficiently small $(\delta=\delta(\sigma, f))$

$$
\underset{t \rightarrow \pm \infty}{s-\lim _{\sigma}} R_{\sigma}^{ \pm}(f) e^{-i t H_{0}}=0
$$
$l \in \mathbf{N}$

(c) for $\delta>0$ and $b>0$ sufficiently small $(\delta=\delta(\sigma, f) ; b=b(\sigma, f))$ and for every

$$
\left\|F(|x|<b|t|) e^{-i t H_{0}} R_{\sigma}^{\mp}(f)\right\|<C_{l}(1+|t|)^{-l} \quad \text { if } \pm t>0
$$

for some constants $C_{l}>0$.

This theorem quantifies results which are intuitively clear. For example when $t>$ 0 (c) says that every freely evolving outgoing state with nonzero velocity $(f(0)=0)$ is predictably far from the origin in the future. See $[\mathbf{1 1}, \mathbf{1 2}]$ for a more thorough discussion. A proof of Theorem 2.2 is given in the Appendix.

3. Abstract existence. In this and the next section we shall prove existence and completeness of the wave operators in an abstract two-Hilbert space setting. Our assumptions are $\left(R_{\sigma}^{\mp}(f)\right.$ were defined in $\left.\S 2\right)$ :

(H1) $H_{0}=-\frac{1}{2} \Delta$ (as in (1.3)) and $H$ is a selfadjoint operator on a Hilbert space H. There exist two bounded linear operators $J^{ \pm}$from $L^{2}\left(\mathbf{R}^{n}\right)$ to $\nVdash$.

Denote the spectral measures of $H$ and $H_{0}$ by $E$ and $E_{0}$.

(H2) $(H-z)^{-1}\left(H J^{ \pm}-J^{ \pm} H_{0}\right) R_{\sigma}^{\mp}(f)$ are compact for some $z \in \mathbf{C} \backslash \mathbf{R}$, every $f \in C_{0}^{\infty}((0, \infty))$ and every $\sigma, \sigma_{0} \leq \sigma<1$, where $\sigma_{0}$ is some constant $-1 \leq \sigma_{0}<0$.

(H3) $\left\|E(I)\left(H J^{ \pm}-J^{ \pm} H_{0}\right) R_{\sigma}^{ \pm}(f) F\left(\left|x^{\prime}\right| \geq r\right)\right\| \in L^{1}([0, \infty) d r)$ for every compact $I \subseteq \mathbf{R}$, every $f \in C_{0}^{\infty}((0, \infty))$ and every $\sigma, \sigma_{0} \leq \sigma<1$.

(H4) $s-\lim _{t \rightarrow \mp \infty} R_{\sigma}^{ \pm}(f)\left[\left(J^{ \pm}\right)^{*} J^{ \pm}-1\right] R_{\sigma}^{ \pm}(f) e^{-i t H_{0}}=0$ for every $\sigma, \sigma_{0} \leq \sigma<1$, and every real $f$ in $C_{0}^{\infty}([0, \infty))$. (Here 1 denotes the identity operator; "**" denotes adjoint.)

(H5) For every compact $I$ in $(0, \infty)$ there is $m_{0} \in \mathbf{N}$ so that

$$
E_{c} E(I)\left[J^{ \pm} f_{m}\left(H_{0}\right)\left(J^{ \pm}\right)^{*}-1\right] E(I) E_{c}
$$

are compact for $m>m_{0}$, where $\left\{f_{m}\right\}_{m \in \mathbf{N}} \in C_{0}^{\infty}((0, \infty))$ and $f_{m}(\lambda)=1$ for $1 / m \leq \lambda<m$ and $f_{m}$ is real. (Here $E_{c}$ is the orthogonal projection of $\forall$ onto the subspace of continuity of $H$.)

(H6) $R_{\sigma_{1}}^{\mp}\left(f_{1}\right)\left(J^{ \pm}\right)^{*} J^{\mp} R_{\sigma_{2}}^{ \pm}\left(f_{2}\right)$ are compact for some $\sigma_{1}, \sigma_{2}, 0<\sigma_{1}, \sigma_{2}<1$, and every $f_{1}, f_{2} \in C_{0}^{\infty}((0, \infty))$.

REMARK. Hypotheses (H1), (H2) and (H3) are the usual assumptions (for the one-Hilbert space short range case) adapted to the present situation. See [14]. We only remark that if (H2) holds for some $z$ it holds for $z$ in $\mathbf{C} \backslash \mathbf{R}$ and that in (H2) and (H3), $H J^{ \pm}-J^{ \pm} H_{0}$ is to be interpreted as the operator associated with the obvious sesquilinear form. We have chosen to work with the operators $\left(H J^{ \pm}-J^{ \pm} H_{0}\right) R_{\sigma}^{ \pm}(f)$ in contrast to Isozaki and Kitada [20] who essentially work with $H J^{ \pm} R_{\sigma}^{ \pm}(f)-J^{ \pm} R_{\sigma}^{ \pm}(f) H_{0}$. Hypotheses (H4), (H5) and (H6) will be discussed in the next section.

The object of this section is to prove the following theorem. 
ThEOREM 3.1. Assume (H1), (H2) and (H3). Then

$$
\Omega^{ \pm}=\underset{t \rightarrow \mp \infty}{s-l i m_{m}} e^{i t H} J^{ \pm} e^{-i t H_{0}}
$$

exist and $\left(\Omega^{ \pm}-J^{ \pm}\right) R_{\sigma}^{ \pm}(f)$ are compact for any $f \in C_{0}^{\infty}((0, \infty))$ and any $\sigma, \sigma>-1$, and $\sigma_{0} \leq \sigma<1\left(\sigma_{0}\right.$ as in $\left.(\mathrm{H} 2)\right)$.

We may write $\Omega^{ \pm}\left(J^{ \pm}\right)$(or $\Omega^{ \pm}\left(H, H_{0} ; J^{ \pm}\right)$) for $\Omega^{ \pm}$when the reference to $J^{ \pm}$ (or $H$ and $H_{0}$ ) needs clarification. The fundamental properties of the two-Hilbert space wave operators $\Omega^{ \pm}$may be found in $[\mathbf{4 2}, \mathrm{pp}$. 34-36]. We require the following "intertwining principle".

LEMMA 3.2. Let $H$ and $H_{0}$ be selfadjoint operators on Hilbert spaces $\sharp$ and $\mathscr{H}_{0}$ and let $E$ and $E_{0}$ be their spectral measures. Let $J$ be a bounded linear operator from $\mathfrak{H}_{0}$ to $\mathscr{H}$. If $\Omega^{ \pm}=\Omega^{ \pm}\left(H, H_{0}, J\right)$ exists, then

$$
\Omega^{ \pm} E_{0}(A)=E(A) \Omega^{ \pm} \quad \text { for any Borel set } A .
$$

For a proof see [42, proof of Proposition 4, p. 34].

In preparation for the proof of Theorem 3.1 we establish the following lemma.

LEMMA 3.3. Assume (H1) and (H2). Then for every $\sigma, \sigma_{0} \leq \sigma<1$, and $f \in C_{0}^{\infty}((0, \infty))$

$$
\lim _{n \rightarrow \infty}\left\|E(\{\lambda:|\lambda|>n\}) J^{ \pm} R_{\sigma}^{ \pm}(f)\right\|=0 .
$$

ProOF. We write

$$
\begin{aligned}
& E(\{\lambda:|\lambda|>n\})(H-z)^{-1}\left(H J^{-}-J^{-} H_{0}\right) R_{\sigma}^{-}(f) \\
& \quad=E(\{\lambda:|\lambda|>n\}) J^{-} R_{0}^{-}(f)-E(\{\lambda:|\lambda|>n\})(H-z)^{-1} J^{-}\left(H_{0}-z\right) R_{\sigma}^{-}(f) .
\end{aligned}
$$

The left side converges to 0 in operator norm because of hypothesis (H2) and because $E(\{\lambda:|\lambda|>n\})$ goes to 0 strongly as $n \rightarrow \infty$. The second term on the right also goes to 0 in operator norm as $n \rightarrow \infty$ because $E(\{\lambda:|\lambda|>n\})(H-z)^{-1}$ does. The remaining term must also go to 0 which establishes the result in the "-" case. The other case is similar.

We now prove the theorem.

PROOF OF THEOREM 3.1. We establish only the outgoing ("-") case, the other being similar. Several simplications can be made. First, to prove the existence of $\Omega^{-}$it clearly suffices to prove the strong convergence of $e^{i t H} J^{-} e^{-i t H_{0}} E_{0}\left(I_{0}\right)$ as $t \rightarrow \infty$ for an arbitrary compact subset $I_{0}$ of $(0, \infty)$. Next it suffices to prove

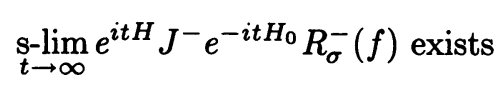

for $f \in C_{0}^{\infty}((0, \infty)), f(\lambda)=1$ if $\lambda \in I_{0}$, and $\sigma_{0} \leq \sigma<1$ and $\sigma>-1$. Suppose $\phi=E_{0}\left(I_{0}\right) \phi$. Then, since $E_{0}\left(I_{0}\right)=\left(R_{\sigma}^{-}(f)+R_{-\sigma}^{+}(f)\right) E_{0}\left(I_{0}\right)$,

$$
\begin{aligned}
e^{i t H} J^{-} e^{-i t H_{0}} \phi= & e^{i s H}\left[e^{i(t-s) H} J^{-} e^{-i(t-s) H_{0}} R_{\sigma}^{-}(f)\right] e^{-i s H_{0}} \phi \\
& +e^{i t H} J^{-} e^{-i(t-s) H_{0}}\left[R_{-\sigma}^{+}(f) e^{-i s H_{0}} \phi\right] .
\end{aligned}
$$

The second term on the right can be made arbitrarily small for all $t$ by choosing $s$ large according to Theorem 2.2(b). For fixed $s$, the first term on the right converges 
as $t \rightarrow \infty$ by (3.1). Thus proving (3.1) will prove $\Omega^{-}$exists. We can further reduce the proof of (3.1) to establishing

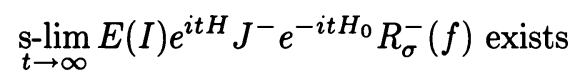

for any compact set $I \subseteq \mathbf{R}$. To show this write

$$
\begin{aligned}
e^{i t H} J^{-} e^{-i t H_{0}} R_{0}^{-}(f)= & e^{i t H}\left[E(\{|\lambda|>n\}) J^{-} R_{\sigma}^{-}\left(f_{1}\right)\right] e^{-i t H_{0}} R_{\sigma}^{-}(f) \\
& +E(\{|\lambda|>n\}) e^{i t H} J^{-}\left[R_{-\sigma}^{+}\left(f_{1}\right) e^{-i t H_{0}}\right] R_{\sigma}^{-}(f) \\
& +E(\{\lambda \leq n\}) e^{i t H} J^{-} e^{-i t H_{0}} R_{\sigma}^{-}(f),
\end{aligned}
$$

where $f_{1} \in C_{0}^{\infty}((0, \infty))$ and $f_{1}(\lambda)=1$ if $\lambda \notin \operatorname{supp}(f)$. Applying Lemma 3.3 to the first term on the right side and Theorem 2.2(b) to the second, we see (3.2) implies (3.1).

Thus establishing (3.2) will prove $\Omega^{-}$exists. We shall actually prove that

$$
E(I)\left[e^{i t H} J^{-} e^{-i t H_{0}}-J^{-}\right] R_{\sigma}^{-}(f)
$$

converges in operator norm as $t \rightarrow \infty$. This will prove (3.2) and that $\Omega^{-}$exists. If we also show that the operators in equation (3.3) are compact, then we will have $E(I)\left[\Omega^{-}-J^{-}\right] R_{\sigma}^{-}(f)$ is compact. This will complete the proof of the theorem because

$$
\begin{aligned}
\left(\Omega^{-}-J^{-}\right) R_{\sigma}^{-}(f)= & E(\{|\lambda| \leq n\})\left[\Omega^{-}-J^{-}\right] R_{\sigma}^{-}(f) \\
& +E(\{|\lambda|>n\}) J^{-} R_{\sigma}^{-}(f)
\end{aligned}
$$

for large $n$, by Lemma 3.2 and so $\left[\Omega^{-}-J^{-}\right] R_{\sigma}^{-}(f)$ is compact, by Lemma 3.3.

Consider therefore the operators in (3.3). Differentiate and integrate (Cook's method)

$$
\begin{aligned}
E(I)\left[e^{i t H} J^{-} e^{-i t H_{0}}-J^{-}\right] R_{\sigma}^{-}(f) & \\
= & i \int_{0}^{t} E(I) e^{i \tau H}\left(H J^{-}-J^{-} H_{0}\right) e^{-i \tau H_{0}} R_{\sigma}^{-}(f) d \tau \\
= & i \int_{0}^{t} E(I) e^{i \tau H}\left(H J^{-}-J^{-} H_{0}\right)\left[R_{-\sigma^{\prime}}^{+}\left(f_{1}\right) e^{-i \tau H_{0}} R_{\sigma}^{-}(f)\right] d \tau \\
& +i \int_{0}^{t} E(I) e^{i \tau H}\left(H J^{-}-J^{-} H_{0}\right) R_{\sigma^{\prime}}^{-}\left(f_{1}\right) e^{-i \tau H_{0}} R_{\sigma}^{-}(f) d \tau,
\end{aligned}
$$

where $f_{1} \in C_{0}^{\infty}((0, \infty))$ and $f_{1}(\lambda)=1$ if $\lambda \notin \operatorname{supp}(f)$, and where $\sigma^{\prime}>\sigma$ and $\sigma_{0} \leq \sigma^{\prime}<1$. Observe that each of the integrands above is a continuous operator valued function of $\tau$ and so the integrals exist as Riemann integrals. Also the last two integrands are compact by (H2) and because $R_{-\sigma^{\prime}}^{+}\left(f_{1}\right) e^{-i t H_{0}} R_{\sigma}^{-}\left(f_{1}\right)$ is by Theorem 2.2(a). Thus the operators in (3.3) are compact. To prove the desired convergence of the operators in (3.3), we need only show that the two integrands are absolutely integrable on $(0, \infty)$. Certainly this is true for the first integrand by Theorem 2.2(a). For the second integrand we have, for any $b>0$,

$$
\begin{aligned}
& \left\|E(I)\left(H J^{-}-J^{-} H_{0}\right) R_{\sigma^{\prime}}^{-}\left(f_{1}\right) e^{-i \tau H_{0}} R_{\sigma}^{-}(f)\right\| \\
& \leq \\
& \quad\left\|E(I)\left(H J^{-}-J^{-} H_{0}\right) R_{\sigma^{\prime}}^{-}\left(f_{1}\right) F(|x| \geq b \tau)\right\|\left\|R_{\sigma}^{-}(f)\right\| \\
& \quad+\left\|E(I)\left(H J^{-}-J^{-} H_{0}\right) R_{\sigma^{\prime}}^{-}\left(f_{1}\right)\right\|\left\|F(|x|<b \tau) e^{-i \tau H_{0}} R_{\sigma}^{-}(f)\right\| .
\end{aligned}
$$


The first term on the right is integrable on $(0, \infty)$ by $(\mathrm{H} 3)$; the second term is integrable by Theorem 2.2 (c) provided $b$ is sufficiently small. It follows that the operators (3.3) converge as $t \rightarrow \infty$ in the operator topology and this establishes the result.

4. Abstract completeness. Now we consider the additional assumptions (H4), (H5) and (H6) introduced in the previous section and derive completeness. In an effort to explain these extra assumptions we analyze them in more depth than is strictly necessary, beginning with (H4).

LEMMA 4.1. Assume (H1) and that $\Omega^{ \pm}=\Omega^{ \pm}\left(H, H_{0} ; J^{ \pm}\right)$exists. Then $\Omega^{ \pm}$is an isometry if and only if, for every real $f \in C_{0}^{\infty}((0, \infty))$,

$$
e^{i t H_{0}} R_{\sigma}^{ \pm}(f)\left[\left(J^{ \pm}\right)^{*} J^{ \pm}-1\right] R_{\sigma}^{ \pm}(f) e^{-i t H_{0}} \rightarrow 0 \quad \text { weakly as } t \rightarrow \mp \infty
$$

for some $\sigma,-1 \leq \sigma<1$. If we further assume (H4), then $\Omega^{ \pm}$are isometries and for every compact interval $I \subseteq(0, \infty)$, every real $f$ in $C_{0}^{\infty}((0, \infty))$ such that $f(\lambda)=1$ if $\lambda \in I$ and every $\sigma, \sigma_{0} \leq \sigma<1$,

$$
\left(\Omega^{ \pm}\right)^{*} E(I)=\underset{t \rightarrow \mp \infty}{\mathrm{s}-\lim _{i}} e^{i t H_{0}} R_{\sigma}^{ \pm}(f)\left(J^{ \pm}\right)^{*} e^{-i t H} E(I) E_{ \pm}
$$

(i.e. the limit exists with the indicated value), where $E_{ \pm}$is the orthogonal projection of $\mathcal{H}$ onto the (closed) range of $\Omega^{ \pm}$.

PROOF. We consider only the "-" (outgoing) case, the incoming case being similar. For each $\phi \in L^{2}\left(\mathbf{R}^{n}\right)$,

$$
\begin{aligned}
\left(e^{i t H_{0}}\right. & \left.R_{\sigma}^{-}(f)\left[\left(J^{-}\right)^{*} J^{-}-1\right] R_{\sigma}^{-}(f) e^{-i t H_{0}} \phi \mid \phi\right) \\
& =\left\|e^{i t H} J^{-} R_{\sigma}^{-}(f) e^{-i t H_{0}} \phi\right\|^{2}-\left\|R_{\sigma}^{-}(f) e^{-i t H_{0}} \phi\right\|^{2} \\
& \rightarrow\left\|\Omega^{-}\left[f\left(H_{0}\right)\right]^{2} \phi\right\|-\left\|\left[f\left(H_{0}\right)\right]^{2} \phi\right\|^{2}
\end{aligned}
$$

as $t \rightarrow \infty$, by Theorem 2.2 (b) and (2.3). Thus $\Omega^{-}$is an isometry if and only if (4.1) holds for all real $f$ in $C_{0}^{\infty}((0, \infty))$.

Now assume (H4) which clearly implies (4.1) so that, $\Omega^{-}$is an isometry. Suppose $\psi=E(I) E_{-} \psi$. Then $\left(\Omega^{-}\right)^{*} \psi=\phi$ for some $\phi$ and $\phi=E_{0}(I) \phi$ by Lemma 3.2. (Note $E_{-}=\Omega^{-}\left(\Omega^{-}\right)^{*}$.) An alternative expression for $\phi$ can be obtained as follows. Choose real $f$ in $C_{0}^{\infty}((0, \infty))$ so that $f(\lambda)=1$ if $\lambda \in I$. Since $\phi=\Omega^{-} \psi$, we have (again by Theorem 2.2(b) and (2.3))

$$
\begin{aligned}
0 & =\lim _{t \rightarrow \infty}\left\|J^{-} R_{\sigma}^{-}(f) e^{-i t H_{0}} \phi-e^{-i t H} \psi\right\| \\
& =\lim _{t \rightarrow \infty}\left\|R_{\sigma}^{-}(f)\left(J^{-}\right)^{*}\left[J^{-} R_{\sigma}^{-}(f) e^{-i t H_{0}} \phi-e^{-i t H} \psi\right]\right\| \\
& =\lim _{t \rightarrow \infty}\left\|\left[R_{\sigma}^{-}(f)\right]^{2} e^{-i t H_{0}} \phi-R_{0}^{-}(f)\left(J^{-}\right)^{*} e^{-i t H} \psi\right\| \\
& =\lim _{t \rightarrow \infty}\left\|e^{-i t H_{0}} \phi-R_{\sigma}^{-}(f)\left(J^{-}\right)^{*} e^{-i t H} \psi\right\|,
\end{aligned}
$$

where we have applied (H4). The result now follows.

Thus (H4) is a slightly stronger hypothesis than is required to assure that $\Omega^{ \pm}$ are isometries. Hypothesis (H4) is convenient because it allows the following reformulation of the completeness postulate which is analogous to that given by Enss in $[11, \S 9]$ for the short range case. 
CoROllary 4.2. Assume (H1) and (H4) and that $\Omega^{ \pm}=\Omega^{ \pm}\left(H, H_{0} ; J^{ \pm}\right)$exists. Let $\sigma$ satisfy $\sigma_{0} \leq \sigma<1, I \subseteq(0, \infty)$ be compact and $f \in C_{0}^{\infty}((0, \infty))$ be real and satisfy $f(\lambda)=1$ if $\lambda \in I$. Suppose $\phi=E(I) E_{c} \phi$. If there is $\left\{t_{j}\right\}_{j \in \mathbf{N}} \subseteq \mathbf{R}$ convergent to $\mp \infty$ as $k \rightarrow \infty$ so that

$$
\lim _{j \rightarrow \infty}\left(\Omega^{ \pm} R_{\sigma}^{ \pm}(f)\left(J^{ \pm}\right)^{*}-1\right) e^{-i t_{j} H} \phi=0,
$$

then $\phi$ belongs to the range $R\left(\Omega^{ \pm}\right)$of $\Omega^{ \pm}$. Conversely (for $\left.\phi=E(I) E_{c} \phi\right)$, if $\phi \in R\left(\Omega^{ \pm}\right)$, then (4.2) holds for every sequence $\left\{t_{j}\right\}_{j \in \mathbf{N}}$, such that $t_{j} \rightarrow \mp \infty$ as $j \rightarrow \infty$.

ProOF. We consider only the outgoing, "-", case. By the intertwining principle Lemma $3.2,(4.2)$ is equivalent to

$$
\lim _{j \rightarrow \infty}\left\|\Omega^{-} e^{i t_{j} H_{0}} R_{\sigma}^{-}(f)\left(J^{-}\right)^{*} e^{-i t_{j} H} \phi-\phi\right\|=0 .
$$

Thus (4.2) implies that the (closed) range of $\Omega^{-}$contains $\phi$. Conversely if $\phi \in$ $R\left(\Omega^{-}\right)$, then by the previous lemma,

$$
\lim _{t \rightarrow \infty} \Omega^{-} e^{i t H_{0}} R_{\sigma}^{-}(f)\left(J^{-}\right)^{*} e^{-i t H} \phi=\Omega^{-}\left(\Omega^{-}\right)^{*} \phi=E_{-} \phi=\phi
$$

which concludes the proof.

We shall now assume (H4) and proceed to consider (H5) and (H6). We shall see that (H5) and (H6) imply (4.2) which is not usually easy to verify directly.

LEMMA 4.3. Assume (H1), (H2), (H3) and (H4), so that $\Omega^{ \pm}=\Omega^{ \pm}\left(H, H_{0} ; J^{ \pm}\right)$ exist. Let $\left\{f_{m}\right\}_{m \in \mathbf{N}} \subseteq C_{0}^{\infty}((0, \infty))$ satisfy $f_{m}(\lambda)=1$ if $-1 / m<\lambda<m$ and $f_{m}$ is real. Let $\sigma$ satisfy $\sigma_{0} \leq \sigma<1, I \subseteq(0, \infty)$ be compact and $\phi=E(I) E_{c} \phi$ and choose $m_{0}$ so that $I \subseteq\left(1 / m_{0}, m_{0}\right)$. Then there is $\left\{t_{j}\right\}_{j=1}^{\infty}$ so that $t_{j} \rightarrow \pm \infty$ and $e^{-i t_{j} H} \phi \rightarrow 0$ weakly as $j \rightarrow \infty$, and for any such sequence $\left\{t_{j}\right\}_{j=1}^{\infty}$, every $m>m_{0}$ and every $\sigma_{1}, \sigma_{2}, 0<\sigma_{1}, \sigma_{2}<-\sigma_{0}$,

$$
\begin{aligned}
\lim _{j \rightarrow \infty}\left(\Omega^{ \pm} R_{\sigma}^{ \pm}\left(f_{m_{0}}\right)\left(J^{ \pm}\right)^{*}-1\right) e^{-i t_{j} H} \phi & \\
=\lim _{j \rightarrow \infty}\left\{\left(J^{ \pm}[\right.\right. & \left.\left.f_{m}\left(H_{0}\right)\right]^{2}\left(J^{ \pm}\right)^{*}-1\right) e^{-i t_{j} H} \phi \\
& +J^{ \pm} R_{\sigma_{1}}^{\mp}\left(f_{m}\right)\left(J^{ \pm}\right)^{*}\left(J^{\mp}\left[f_{m}\left(H_{0}\right)\right]^{2}\left(J^{\mp}\right)^{*}-1\right) e^{-i t_{j} H} \phi \\
& \left.\quad+J^{ \pm} R_{\sigma_{1}}^{\mp}\left(f_{m}\right)\left(J^{ \pm}\right)^{*} J^{\mp} R_{\sigma_{2}}^{ \pm}\left(f_{m}\right)\left(J^{\mp}\right)^{*} e^{-i t_{j} H} \phi\right\}
\end{aligned}
$$

(i.e. one limit exists if and only if the other does). In particular $\phi \in R\left(\Omega^{ \pm}\right)$if and only if one (hence both) of the above limits exists and is 0.

We leave aside the proof of Lemma 4.3 until the end of this section. Hypotheses (H5) and (H6) are formulated so as to conveniently verify that the limit in Lemma 4.3 exists.

Corollary 4.4. Assume (H1), (H2), (H3), (H4), (H5) and (H6). Then the wave operators $\Omega^{ \pm}\left(H, H_{0} ; J^{ \pm}\right)$exist and are isometries with range $E([0, \infty)) E_{c} H$.

REMARKS. 1. Hypotheses (H4) and (H6) are trivial when $J^{+}=J^{-}=1$ (and $H=L^{2}\left(\mathbf{R}^{n}\right)$ ) but (H5) is not. Hypothesis (H5) becomes

$$
E_{c} E(I)\left[E_{0}((0,1 / m))+E_{0}((m, \infty))\right] E(I) E_{c}
$$


is compact for $m$ large enough, which is certainly the case if $(H-z)^{-1}-\left(H_{0}-z\right)^{-1}$ is compact. (See [14] for further discussion.)

2. Usually completeness results conclude that the ranges of $\Omega^{ \pm}$are $E_{c} H$. Corollary 4.4 has a weaker conclusion and the reader can easily check that the hypotheses may allow $E_{0} E((-\infty, 0)) \neq 0$. To exclude this eventuality an additional hypothesis is required, such as the essential spectrum of $H$ is $[0, \infty)$.

3. Hypotheses (H5) and (H6) could be slightly weakened in Corollary 4.4 by assuming the right side of $(4.3)$ is 0 .

PROOF OF COROLLARY 4.4. The existence of $\Omega^{ \pm}$is guaranteed by Theorem 3.1 and $\Omega^{ \pm}$are isometries by Lemma 4.1. We prove that the range $R\left(\Omega^{-}\right)$of $\Omega^{-}$ is $E([0, \infty)) E_{c} \sharp$, the proof for $R\left(\Omega^{+}\right)$is similar. Since $R\left(\Omega^{-}\right) \subseteq E([0, \infty)) E_{c} \nLeftarrow$ in general (by Lemma 3.2) and $R\left(\Omega^{-}\right)$is closed, it suffices to show that if $\phi=E(I) E_{c} \phi$ for some compact $I \subseteq(0, \infty)$, then $\phi \in R\left(\Omega^{-}\right)$. To show this, apply Lemma 4.3. We check that the expression on the right side of (4.3) is 0. By (H5) and (H6) this expression is of the form $\lim _{j \rightarrow \infty} K e^{-i t_{j} H} \phi$, where $K$ is compact. Since $e^{-i t_{j} H} \phi \rightarrow 0$ weakly as $j \rightarrow \infty$ the result follows.

It remains to establish Lemma 4.3.

PROOF OF LEMMA 4.3. The existence of a sequence $\left\{t_{j}\right\}_{j=1}^{\infty}$ so that $e^{-i t_{j} H} \phi \rightarrow$ 0 weakly follows from Wiener's theorem $[42$, p. 340] and the spectral representation of $\left(e^{-i t H} \phi \mid \phi\right)$. For the remainder of the proof we consider only the outgoing, "-", case; the incoming case is similar. We have seen already, in Theorem 3.1 , that $\Omega^{-}$ exists and that $\left(\Omega^{-}-J^{-}\right) R_{\sigma}^{-}\left(f_{m}\right)$ is compact. We shall use this compactness to show that on the left side of equation (4.3) we may replace $m_{0}$ and $\sigma$ by arbitrary $m \geq m_{0}$ and $\sigma_{1}, \sigma_{0} \leq \sigma_{1}<1$. Indeed the weak convergence of $e^{-i t_{j} H} \phi$ implies $R_{\sigma}^{-}\left(f_{m}\right)\left(\Omega^{-}-J^{-}\right)^{*} e^{-i t_{j} H} \phi$ goes to 0 as $k \rightarrow \infty$ so that

$$
\begin{aligned}
\lim _{j \rightarrow \infty} \Omega^{-} R_{\sigma}^{-}\left(f_{m_{0}}\right)\left(J^{-}\right)^{*} e^{-i t_{j} H} \phi-\Omega^{-} R_{\sigma_{1}}^{-}\left(f_{m}\right)\left(J^{-}\right)^{*} e^{-i t_{j} H} \phi \\
=\lim _{j \rightarrow \infty} \Omega^{-}\left\{R_{\sigma}^{-}\left(f_{m_{0}}\right)-R_{\sigma_{1}}^{-}\left(f_{m}\right)\right\}\left(\Omega^{-}\right)^{*} e^{-i t_{j} H} \phi \\
=\lim _{j \rightarrow \infty}\left[\Omega^{-}\left\{\left[f_{m_{0}}\left(H_{0}\right)\right]^{2}-\left[f_{m}\left(H_{0}\right)\right]^{2}\right\} E_{0}(I) e^{-t_{j} H_{0}}\left(\Omega^{-}\right)^{*} \phi\right. \\
\left.\quad-\Omega^{-}\left\{R_{-\sigma}^{+}\left(f_{m_{0}}\right) e^{-i t_{j} H_{0}}-R_{-\sigma_{1}}^{+}\left(f_{m}\right) e^{-i t_{j} H_{0}}\right\}\left(\Omega^{-}\right)^{*} \phi\right]
\end{aligned}
$$

by Lemma 3.2 and (2.3). This limit is 0 , because $\left\{\left[f_{m_{0}}\left(H_{0}\right)\right]^{2}-\left[f_{m}\left(H_{0}\right)\right]^{2}\right\} E_{0}(I)=$ 0 and because of Theorem 2.2(b). Therefore to prove (4.3) we may replace $m_{0}$ and $\sigma$ by any $m \geq m_{0}$ and $\sigma_{1}, \sigma_{0} \leq \sigma_{1}<1$. By the compactness of $\left(\Omega^{-}-J^{-}\right) R_{-\sigma_{1}}^{-}\left(f_{m}\right)$, if $\sigma_{0}<-\sigma_{1}<1$,

$$
\begin{gathered}
\lim _{j \rightarrow \infty}\left(\Omega^{-} R_{-\sigma_{1}}^{-}\left(f_{m}\right)\left(J^{-}\right)^{*}-1\right) e^{-i t_{j} H} \phi=\lim _{j \rightarrow \infty}\left(J^{-} R_{-\sigma_{1}}^{-}\left(f_{m}\right)\left(J^{-}\right)^{*}-1\right) e^{-i t_{j} H} \phi \\
=\lim _{j \rightarrow \infty}\left\{\left(J^{-}\left[f_{m}\left(H_{0}\right)\right]^{2}\left(J^{-}\right)^{*}-1\right) e^{-i t_{j} H} \phi-J^{-} R_{\sigma_{1}}^{+}\left(f_{m}\right)\left(J^{-}\right)^{*} e^{-i t_{j} H} \phi\right\} \\
=\lim _{j \rightarrow \infty}\left\{\left(J^{-}\left[f_{m}\left(H_{0}\right)\right]^{2}\left(J^{-}\right)^{*}-1\right) e^{-i t_{j} H} \phi\right. \\
\quad+J^{-} R_{\sigma_{1}}^{+}\left(f_{m}\right)\left(J^{-}\right)^{*}\left(J^{+}\left[f_{m}\left(H_{0}\right)\right]^{2}\left(J^{+}\right)^{*}-1\right) e^{-i t_{j} H} \phi \\
-J^{-} R_{\sigma_{1}}^{+}\left(f_{m}\right)\left(J^{-}\right)^{*} J^{+} R_{\sigma_{2}}^{-}\left(f_{m}\right)\left(J^{+}\right)^{*} e^{-i t_{j} H} \phi \\
\left.-J^{-} R_{\sigma_{1}}^{+}\left(f_{m}\right)\left(J^{-}\right)^{*} J^{+} R_{-\sigma_{2}}^{+}\left(f_{m}\right)\left(J^{+}\right)^{*} e^{-i t_{j} H} \phi\right\}
\end{gathered}
$$


if $\sigma_{0}<-\sigma_{1}<1$, and for any $\sigma_{2}, \sigma_{0} \leq-\sigma_{2}<1$. This will prove equation (4.3), if we show that the final term above is 0 in the limit. To do this we again use $R_{\sigma_{2}}^{-}\left(f_{m}\right)\left(\Omega^{-}-J^{-}\right)^{*} e^{-i t_{j} H} \phi \rightarrow 0$ as $j \rightarrow \infty$, so that

$$
\lim _{j \rightarrow \infty} R_{-\sigma_{2}}^{+}\left(f_{m}\right)\left(J^{-}\right)^{*} e^{-i t_{j} H} \phi=\lim _{j \rightarrow \infty} R_{-\sigma_{2}}^{+}\left(f_{m}\right)\left(\Omega^{-}\right)^{*} e^{-i t_{j} H} \phi=0
$$

by the intertwining principle (Lemma 3.2) and Theorem 2.2(b). This proves (4.3). The final conclusion is an immediate consequence of Corollary 4.2 and the proof is complete.

5. The operators $J^{ \pm}$. In this section and the next, we establish a special case of Theorem 1.1, the case $U=0$ which is the one considered in [20] by Isozaki and Kitada. To do so, we naturally wish to apply Corollary 4.4 which requires choosing $J^{ \pm}$. Following [20], we specify $J^{ \pm}$to be of the form

$$
J^{ \pm} \phi(x)=\int_{\mathbf{R}^{n}} e^{i x \cdot \xi+i \theta^{ \pm}(x, \xi)} \hat{\phi}(\xi) d_{1} \xi \quad\left(\phi \in S\left(\mathbf{R}^{n}\right)\right)
$$

for some $\theta^{ \pm}$. (Here $d_{1} \xi=(2 \pi)^{-n / 2} d \xi$.) If $\theta^{ \pm}=0$, then $J^{ \pm}$are both the identity on $L^{2}\left(\mathbf{R}^{n}\right)$ by Plancherel's theorem and so if $\theta^{ \pm}$are small in some sense then it is reasonable to hope that $J^{ \pm}$are bounded and satisfy hypotheses (H4), (H5) and (H6) of $\S 3$. Thus the crucial hypotheses are (H2) and (H3) which essentially require that $(H-z)^{-1}\left[H J^{ \pm}-J^{ \pm} H_{0}\right] R_{\sigma}^{ \pm}(f)$ be small. Recalling that $H=H_{0}+V+V_{s}$ and $H_{0}=-\frac{1}{2} \Delta$, we have at least formally that

$$
\begin{aligned}
& \left(H J^{-}-J^{-} H_{0}\right) R_{\sigma}^{-}(f) \phi(x)=V_{s} J^{-} R_{\sigma}^{-}(f) \phi(x) \\
& +\int_{\mathbf{R}^{n}} e^{i x \cdot \xi+i \theta^{-}(x, \xi)}\left\{\frac{1}{2}\left|\xi+\nabla_{x} \theta^{-}(x, \xi)\right|^{2}-\frac{1}{2}|\xi|^{2}\right. \\
& \left.\quad+V(x)-\frac{i}{2} \Delta_{x} \theta^{-}(x, \xi)\right\}\left(R_{\sigma}^{-}(f) \phi\right)(\xi) d_{1} \xi
\end{aligned}
$$

(and similarly when "+" replaces "-"), where $\Delta_{x}$ is the Laplacian in the $x \in \mathbf{R}^{n}$ variable (and similarly for $\nabla_{x}$ ). Presumably the first term on the right of (5.2) will be suitably small simply because $V_{s}$ is short range and thus it is the integral expression that is important. If we restrict $\theta^{-}$to be real, as we shall until $\S 7$, then the optimal choice of $\theta^{-}$will satisfy the eikonal equation

$$
\frac{1}{2}\left|\xi+\nabla_{x} \theta^{-}(x, \xi)\right|^{2}+V(x)=\frac{1}{2}|\xi|^{2}
$$

when $(x, \xi) \in M_{\sigma}^{-}$(see (2.1)) and of course $\Delta_{x} \theta^{-}$must also be small. The existence of $\theta^{-}$with the requisite properties is guaranteed by the following result.

THEOREM 5.1 (ISOZAKI AND KITADA). Suppose $V$ satisfies (LR1). Then there exist real valued functions $\theta_{1}^{ \pm}$in $C^{\infty}\left(\mathbf{R}^{2 n}\right)$ such that

$$
\theta_{1}^{+}(x, \xi)=-\theta_{1}^{-}(x,-\xi)
$$

and for any $d>0$ and $\sigma_{1},-\frac{1}{2}<\sigma_{1}<0$, there is $R>0$ so that for all $(\lambda, \xi)$ in $\left\{(\lambda, \xi) \in \mathbf{R}^{2 n}: \mp x \cdot \xi>2 \sigma_{1}|x||\xi| ;|\xi|>d ;|x|>R\right\}$

$$
\frac{1}{2}\left|\xi+\nabla_{x} \theta_{1}^{ \pm}(x, \xi)\right|^{2}+V(x)=\frac{1}{2}|\xi|^{2}
$$

and

$$
\left|D_{x}^{\alpha} D_{\xi}^{\beta} \theta_{1}^{ \pm}(x, \xi)\right| \leq C_{\alpha, \beta, d}|\xi|^{-1}\langle x\rangle^{1-|\alpha|-\varepsilon_{0}}
$$

for all multi-indices $\alpha, \beta \in \mathbf{N}^{n}$. Here $\varepsilon_{0}>0$ is the constant in (LR1). 
For a proof of this result see [20, Proposition 2.4] (where $\theta_{1}^{ \pm} x, \xi$ are denoted $\left.\phi_{ \pm}(x, \xi)-x \cdot \xi\right)$. Although we shall not repeat it here, the proof itself is of considerable interest. It involves computing the action along appropriate classical paths and is based on an earlier construction $[18,27-30]$ of a solution of Hamilton's equation.

The functions $\theta^{ \pm}$of (5.1) are obtained by cutting off $\theta_{1}^{ \pm}$appropriately. To do so, first choose a strictly decreasing sequence $\left\{d_{j}\right\}_{j=0}^{\infty}$ so that $d_{0}=1$ and $\lim _{j \rightarrow \infty} d_{j}=$ 0. Define $\left\{U_{j}\right\}_{j=0}^{\infty}$ by

$$
\begin{gathered}
U_{0}=\left\{\xi \in \mathbf{R}^{n}:|\xi|>d_{1}\right\} \\
U_{j}=\left\{\xi \in \mathbf{R}^{n}: d_{j+1}<|\xi|<d_{j-1}\right\} \quad \text { if } j>0
\end{gathered}
$$

so that $\left\{U_{j}\right\}_{j=0}^{\infty}$ is an open cover of $\mathbf{R}^{n} \backslash\{0\}$. Choose a $C^{\infty}$ partition of unity $\left\{\mu_{j}\right\}_{j=0}^{\infty}$ corresponding to this cover (i.e. $\mu_{j} \in C^{\infty}\left(\mathbf{R}^{n}\right)$ is nonnegative and supported in $U_{j}$ and $\sum_{j} \mu_{j}(\xi)=1$ if $\left.\xi \neq 0\right)$. We shall also specify that $\mu_{j}(\xi)=\mu_{j}(-\xi)$. Next choose real $g_{1}$ in $C^{\infty}\left(\mathbf{R}^{n}\right)$ so that $g_{1}(x)=1$ if $|x|>2$ and $g_{1}(x)=0$ if $|x|<1$. Choose $\sigma_{1},-\frac{1}{2}<\sigma_{1}<0$, and $\chi \in C^{\infty}([-1,1])$ so that $\chi(r)=0$ if $r<2 \sigma_{1}, \chi(r)=1$ if $r>\sigma_{1}$ and $\chi$ is real valued. Define

$$
\theta^{ \pm}(x, \xi)=\left\{\begin{array}{l}
\theta_{1}^{ \pm}(x, \xi) \chi\left(\mp \frac{x \cdot \xi}{|x||\xi|}\right) \sum_{j=0}^{\infty} g_{1}\left(x / R_{j}\right) \mu_{j}(\xi) \text { if } x, \xi \neq 0 \\
0 \quad \text { if } x=0 \text { or } \xi=0
\end{array}\right.
$$

where $\left\{R_{j}\right\}_{j=0}^{\infty}$ is a strictly increasing sequence of positive numbers which will be specified in the following lemma. Since $\mu_{j}(\xi)=\mu_{j}(-\xi), \theta^{ \pm}$have the same symmetry as $\theta_{1}^{ \pm}$:

$$
\theta^{+}(x, \xi)=-\theta^{-}(x,-\xi)
$$

Intuitively $\theta^{ \pm}$is $\theta_{1}^{ \pm}$cut off outside $M_{\sigma_{1}}^{ \pm}$and further restricted so that when the velocity $\xi$ is small the position $x$ is far from the origin and this is of course the region where one would hope to be able to approximate the wave operators.

LEMMA 5.2. Assume $V$ satisfies (LR1). Let $\eta>0, m_{0} \in \mathbf{N}, m_{0}>1$ and $\varepsilon_{1}$, $0<\varepsilon_{1}<\varepsilon_{0}$, be given ( $\varepsilon_{0}$ as in (LR1)). Then there is a strictly increasing sequence $\left\{R_{j}\right\}_{j \geq 0}$ so that $R_{0}>1$ and $\lim _{j \rightarrow \infty} R_{j}=\infty$ so that $\theta^{ \pm}$defined by (5.4) belongs to $C^{\infty}\left(\mathbf{R}^{n}\right)$ and

$$
\left|D_{x}^{\alpha} D_{\xi}^{\beta} \theta^{ \pm}(x, \xi)\right|<\eta\langle\xi\rangle^{-1}\langle x\rangle^{1-|\alpha|-\varepsilon_{1}} \quad \text { for all }(x, \xi) \in \mathbf{R}^{2 n}
$$

and all multi-indices $\alpha, \beta \in \mathbf{N}^{n},|\alpha|,|\beta| \leq m_{0}$. Also, for any $d>0$

$$
\left|D_{x}^{\alpha} D_{\xi}^{\beta} \theta^{ \pm}(x, \xi)\right|<C_{\alpha, \beta, d}\langle\xi\rangle^{-1}\langle x\rangle^{1-|\alpha|-\varepsilon_{0}} \quad \text { for all }(x, \xi) \in \mathbf{R}^{2 n},|\xi|>d
$$

and all multi-indices $\alpha, \beta \in \mathbf{N}^{n}$. Moreover if $|\xi|>d_{j}$ and $|x|>2 R_{j}$ for some $j \in \mathbf{N}$ and $\mp x \cdot \xi>\sigma_{1}|x||\xi|$, then

$$
\frac{1}{2}\left|\xi+\nabla_{x} \theta^{ \pm}(x, \xi)\right|^{2}+V(x)=\frac{1}{2}|\xi|^{2} .
$$

ProOF. Observe that $\theta^{ \pm}(x, \xi)=0$ whenever $|x|<R_{0}$ or for some $j,|\xi|<d_{j}$ and $|x|<R_{j}$. Using this, one easily checks that $\theta^{ \pm} \in C^{\infty}\left(\mathbf{R}^{2 n}\right)$. Also $\theta^{ \pm}(x, \xi)=$ $\theta_{1}^{ \pm}(x, \xi)$ if $(x, \xi) \in\left\{(x, \xi): \mp x \cdot \xi>\sigma_{1}|x||\xi|,|\xi|>d_{j},|x|>2 R_{j}\right\}$ for some $j$. 
By Theorem 5.1, it follows that $\theta^{ \pm}$satisfies the eikonal equation (5.3) on this set, provided we choose $R_{j}$ sufficiently large.

It remains to establish (5.6) and (5.7). Since $\theta^{ \pm}(x, \xi)$ is 0 along with all its derivatives if $|x|<R_{0}$ or $\xi=0$, we may suppose $x \neq 0$ and $\xi \neq 0$. In this case, one easily checks that, for any multi-indices $\alpha, \beta \in \mathbf{N}^{n}$

$$
\left|D_{x}^{\alpha} D_{\xi}^{\beta} \chi\left(\mp \frac{x \cdot \xi}{|x||\xi|}\right)\right| \leq C_{\alpha, \beta}|x|^{-|\alpha|}|\xi|^{-|\beta|}, \quad x \neq 0, \xi \neq 0 .
$$

Also, if $\xi \neq 0$, there is $k \in \mathbf{N}$ so that $d_{k-1}>|\xi| \geq d_{k}\left(d_{-1}=\infty\right)$. Therefore, for any $\alpha, \beta \in \mathbf{N}^{n}$

$$
\begin{aligned}
\left|D_{x}^{\alpha} D_{\xi}^{\beta} \sum_{j=0}^{\infty} g_{1}\left(x / R_{j}\right) \mu_{j}(\xi)\right| & =\left|\sum_{j=k-1}^{k} R_{j}^{-|\alpha|}\left(D_{x}^{\alpha} g_{1}\right)\left(x / R_{j}\right) D_{\xi}^{\beta} \mu_{j}(\xi)\right| \\
& \leq C_{\alpha}\langle x\rangle^{-|\alpha|} \sup \left\{\left|D_{\xi}^{\beta} \mu_{j}(\xi)\right|: \xi \in \mathbf{R}^{n}, j \in\{k-1, k\}\right\} .
\end{aligned}
$$

Here we have noted $C^{-1}<R_{j}\langle x\rangle^{-1}<C(C>0)$ when $\left(D^{\alpha} g_{1}\right)\left(x / R_{j}\right) \neq 0$. Combining these last two estimates with the bound for the derivatives of $\theta_{1}^{ \pm}$in Theorem 5.1 will prove (5.6) and (5.7). More precisely, for any integer $m$, and all $\alpha, \beta \in \mathbf{N}^{n},|\alpha|,|\beta| \leq m$, and all $|\xi|, d_{k-1}>|\xi| \geq d_{k}$, we have by Leibniz's formula $\left|D_{x}^{\alpha} D_{\xi}^{\beta} \theta^{ \pm}(x, \xi)\right|<C_{m} \max C_{\alpha^{\prime}, \beta^{\prime}, d_{j}} \sup \left|D_{\xi}^{\beta^{\prime}} u_{j}(\xi)\right| d_{k}^{-m} R^{-\left(\varepsilon_{0}-\varepsilon_{1}\right)}\langle x\rangle^{1-|\alpha|-\varepsilon_{1}}\langle\xi\rangle^{-1}$, where the maximum is over $\left|\alpha^{\prime}\right|,\left|\beta^{\prime}\right| \leq m$ and $j \in\{k-1, k\}$, the supremum is over $\beta^{\prime},\left|\beta^{\prime}\right| \leq m_{0}, j \in\{k-1, k\}$ and $\xi \in \mathbf{R}^{n}$, and $C_{\alpha^{\prime}, \beta^{\prime}, d}$ is the constant of Theorem 5.1. If we choose $\varepsilon_{1}=\varepsilon_{0}$ then we have (5.7). If however $0<\varepsilon_{1}<\varepsilon_{0}$ and $m=m_{0}$ then we have (5.6) provided $R_{k-1}$ is chosen so large that

$$
C_{m_{0}} \max C_{\alpha^{\prime}, \beta^{\prime}, d_{j}} \sup \left|D_{\xi}^{\beta^{\prime}} u_{j}(\xi)\right| d_{k}^{-m} R_{k-1}^{-\left(\varepsilon_{0}-\varepsilon_{1}\right)}<\eta .
$$

We shall suppose in future that $\theta^{ \pm}$have been completely specified and they satisfy Lemma 5.2 for some fixed $\varepsilon_{1}, 0<\varepsilon_{1}<\varepsilon_{0}, \eta>0$ suitably small and $m_{0}$ suitably large $\left(\eta<1 / 10 ; m_{0}>7 n+6\right.$ will do). For this choice of $\theta^{ \pm}$, define $J^{ \pm}$by (5.1). Two elementary properties of $J^{ \pm}$deserve mention. First, by (5.5)

$$
\overline{J+\bar{\phi}}=J^{-} \phi \quad \text { for all } \phi \in S\left(\mathbf{R}^{n}\right)
$$

(bar denotes complex conjugation). Secondly

$$
J^{ \pm} \phi \in S\left(\mathbf{R}^{n}\right) \quad \text { if } \phi \in\left(\mathbf{R}^{n}\right) \text { and } \phi(\xi)=0 \text { when }|\xi|<d
$$

for some $d>0$. To prove (5.9), write $e^{i x \cdot \xi}=\langle x\rangle^{-2 N}\left(1-\Delta_{\xi}\right)^{N} e^{i x \cdot \xi}$ for any $N \in \mathbf{N}$ so that

$$
J^{ \pm} \phi(x)=\langle x\rangle^{-2 N} \int_{\mathbf{R}^{n}} e^{i x \cdot \xi}\left(1-\Delta_{\xi}\right)^{N}\left\{\varepsilon^{i \theta^{ \pm}(x, \xi)} \phi(\xi)\right\} d \xi .
$$

This and (5.7) imply that $\left|J^{ \pm} \phi(x)\right|<C_{N}\langle x\rangle^{-2 N \varepsilon_{0}}$. Similar reasoning applies to the derivatives of $J^{ \pm} \phi$ and this proves (5.9).

Next we shall prove that $J^{ \pm}$are bounded. Since we will later need to consider the boundedness of the operator (5.2) it is worthwhile to prove a general result similar to Lemma 3.3, part (i) of [20]. 
LemMA 5.3. (i) Suppose $p \in C^{\infty}\left(\mathbf{R}^{3 n}\right), q \in C^{\infty}\left(\mathbf{R}^{2 n}\right)$ and

$$
\begin{gathered}
\max _{|\alpha|,|\beta|,|\gamma|<m_{0}} \sup _{(x, y, \varepsilon) \in \mathbf{R}^{3 n}}\left|D_{x}^{\alpha} D_{y}^{\beta} D_{\xi}^{\gamma} p(x, y, \xi)\right| \equiv|p|_{0}<\infty, \\
\max _{|\alpha|,|\beta|<m_{0}} \sup _{(x, \xi) \in \mathbf{R}^{2 n}}\left|D_{x}^{\alpha} D_{\xi}^{\beta} q(x, \xi)\right| \equiv|q|_{0}<\infty,
\end{gathered}
$$

where $m_{0} \geq 7 n+6$. Then the operators $P^{ \pm}$and $Q^{ \pm}$defined on $S\left(\mathbf{R}^{n}\right)$ by

$$
\begin{gathered}
P^{ \pm} \phi(x)=\int_{\mathbf{R}^{n}}\left\{\int_{\mathbf{R}^{n}} e^{i \xi \cdot(x-y)} e^{i\left(\theta^{ \pm}(x, \xi)-\theta^{ \pm}(y, \xi)\right)} p(x, y, \xi) \phi(y) d y\right\} d \xi \\
Q^{ \pm} \psi(x)=\int_{\mathbf{R}^{n}} e^{i(x \cdot \xi)} e^{i \theta^{ \pm}(x \cdot \xi)} q(x, \xi) \psi(\xi) d \xi
\end{gathered}
$$

are well defined (the integration for $P^{ \pm}$performed in the indicated order) and extend to bounded operators on $L^{2}\left(\mathbf{R}^{n}\right)$ with operator norms

$$
\left\|P^{ \pm}\right\|<c|p|_{0} ; \quad\left\|Q^{ \pm}\right\|<c|q|_{0}
$$

for some $c>0$ not depending on $p$ or $q$.

(ii) $J^{ \pm}$defined on $S\left(\mathbf{R}^{n}\right)$ by (5.1) extend to bounded linear operators on $L^{2}\left(\mathbf{R}^{n}\right)$ and for any $f \in C_{0}^{\infty}(\mathbf{R}), J^{ \pm} f\left(H_{0}\right)\left(J^{ \pm}\right)^{*}-f\left(H_{0}\right)$ are compact operators.

PROOF. We consider only the "-" case; the "+" case is similar (or use (5.5)).

(i) To prove $Q^{-}$is bounded it suffices to prove $\left(Q^{-}\right)^{*}$ is bounded or that $Q^{-}\left(Q^{-}\right)^{*}$ is bounded. We have

$$
Q^{-}\left(Q^{-}\right)^{*} \phi(x)=\int_{\mathbf{R}^{n}} \int_{\mathbf{R}^{n}} e^{i \xi \cdot(x-y)} e^{i\left(\theta^{-}(x, \xi)-\theta^{-}(y, \xi)\right)} q(x, \xi) \overline{q(y, \xi)} \phi(y) d y d \xi .
$$

Therefore, once we have proved that $\left\|P^{-}\right\|<c|p|_{0}$ we will have $\left\|Q^{-}\left(Q^{-}\right)^{*}\right\|<c|p|_{0}$, where $p(x, y, \xi)=q(x, \xi) \overline{q(y, \xi)}$. Since $|p|_{0}<c^{\prime}|q|_{0}^{2}$ we need only prove $\left\|P^{-}\right\|<$ $c|p|_{0}$ to prove (i).

To check that $P^{-}$is well defined, write $e^{-i y \cdot \xi}$ as $\langle\xi\rangle^{-2 N}\left(1-\Delta_{y}\right) e^{-i y \cdot \xi}$ and integrate by parts:

$$
\begin{array}{r}
P^{-} \phi(x)=\int_{\mathbf{R}^{n}}\langle\xi\rangle^{-2 N} \int_{\mathbf{R}^{n}} e^{i \xi \cdot(x-y)}\left(1-\Delta_{y}\right)^{N}\left\{e^{i\left(\theta^{-}(x, \xi)-\theta^{-}(y, \xi)\right)}\right. \\
\cdot p(x, y, \xi) \phi(y)\} d y d \xi .
\end{array}
$$

Since the $y$ derivatives of $e^{-\theta^{-}(y, \xi)} p(x, y, \xi)$ are bounded (by (5.6)), the integral over $\mathbf{R}^{2 n}$ exists if $2 N>n-1$.

To prove $P^{-}$is bounded we shall change variables and then apply the CalderónVaillancourt theorem [2]. Technically to justify each step, we treat the integral defining $P^{-}$as an oscillatory integral.

To make this argument precise, begin by writing

$$
\theta^{-}(x, \xi)-\theta^{-}(y, \xi)=\int_{0}^{1} \nabla_{x} \theta^{-}(y+s(x-y), \xi) d s \cdot(x-y)
$$

Thus, the appropriate change of variables to make $P^{-}$a pseudodifferential operator is

$$
\xi^{\prime}=\Phi(\xi, x, y)=\xi+\int_{0}^{1} \nabla_{x} \theta^{-}(y+s(x-y), \xi) d s .
$$


Using (5.6) it is not difficult to check that, for some $C>0$,

$$
\left|D_{x}^{\alpha} D_{y}^{\beta} D_{\xi}^{\gamma}(\Phi(\xi, x, y)-\xi)\right|<C \eta\langle\xi\rangle^{-1}\left(1+|x|^{2}+|y|^{2}\right)^{-\varepsilon_{1} / 4}
$$

for all multi-indices $\alpha, \beta, \gamma$, where $|\alpha+\beta|,|\gamma| \leq m_{0}$. Also, by (5.6) we see that $\xi \rightarrow \Phi(\xi, x, y)$ has an inverse on all of $\mathbf{R}^{n}$ provided $\eta$ is small enough. Denoting the inverse by $\Psi\left(\xi^{\prime}, x, y\right)$ we have

$$
\left|D_{x}^{\alpha} D_{y}^{\beta} D_{\xi^{\prime}}^{\gamma}\left(\Psi\left(\xi^{\prime}, x, y\right)-\xi^{\prime}\right)\right|<C_{\alpha, \beta, \gamma}\langle\xi\rangle^{-1}\left(1+|x|^{2}+|y|^{2}\right)^{-\varepsilon_{1} / 4}
$$

for all multi-indices $\alpha, \beta, \gamma$ so that $|\alpha|,|\beta|,|\gamma|<\frac{1}{2} m_{0}$ say.

To circumvent integrability problems introduce a cut-off as follows. Choose $g \in C_{0}^{\infty}\left(\mathbf{R}^{n}\right), g(\xi)=1$ if $|\xi|<1$ and $g(\xi)=0$ if $|\xi|>2$. Then

$$
\begin{array}{r}
P^{-} \phi(x)=\lim _{\varepsilon \rightarrow 0+} \int_{\mathbf{R}^{n}} \int_{\mathbf{R}^{n}} e^{i(x-y) \cdot \Phi(\xi, x, y)} g(\varepsilon \Phi(\xi, x, y)) p(x, y, \xi) \phi(y) d y d \xi \\
=\lim _{\varepsilon \rightarrow 0+} \int_{\mathbf{R}^{n}} \int_{\mathbf{R}^{n}} e^{i(x-y) \cdot \xi^{\prime}} g\left(\varepsilon \xi^{\prime}\right) p\left(x, y, \Psi\left(\xi^{\prime}, x, y\right)\right) \\
\cdot\left|\operatorname{det} \partial_{\xi} \Psi\left(\xi^{\prime}, x, y\right)\right| \phi(y) d y d \xi^{\prime} .
\end{array}
$$

Now apply the Calderón-Vaillancourt theorem [2]. Since

$$
\left|D_{x}^{\alpha} D_{y}^{\beta} D_{\xi^{\prime}}^{\gamma} g\left(\varepsilon \xi^{\prime}\right) p\left(x, y, \Psi\left(\xi^{\prime}, x, y\right)\right) \operatorname{det} \partial_{\xi^{\prime}} \Psi\left(\xi^{\prime}, x, y\right)\right|<C_{\alpha, \beta, \gamma, \Psi}\left\langle\xi^{\prime}\right\rangle^{-1}|p|_{0}
$$

for all $|\alpha|,|\beta|,|\gamma|<\frac{1}{2} m_{0}$ and all $\varepsilon, 0<\varepsilon<1$, it follows that $P^{-1}$ is bounded and $\|P\|<c|p|_{0}\left(c\right.$ may depend on $\left.\theta^{-}\right)$.

(ii) Clearly the boundedness of $Q^{-}$implies the boundedness of $J^{-}$. The operator $J^{-} f\left(H_{0}\right)\left(J^{-}\right)^{*}$ is the special case of $P^{-}$when $p(x, y, \xi)=(2 \pi)^{-n} f\left(|\xi|^{2}\right)$. We use the expression for $P^{-}$obtained above but no cut-off is needed this time:

$$
\begin{aligned}
J^{-} f\left(H_{0}\right)\left(J^{-}\right)^{*} \phi-f\left(H_{0}\right) \phi & \int_{\mathbf{R}^{n}} \int_{\mathbf{R}^{n}} e^{i(x-y) \cdot \xi} \\
= & \int_{\mathbf{R}^{n}} \int_{\mathbf{R}^{n}} e^{i(x-y) \cdot \xi} g(\varepsilon x) g(\varepsilon y) \\
& +\int_{\mathbf{R}^{n}} \int_{\mathbf{R}^{n}} e^{i(x-y) \cdot \xi}\{1-g(\varepsilon x) g(\varepsilon y)\} \\
& \cdot\left\{f\left(|\Psi(\xi, x, y)|^{2}\right)\left|\operatorname{det} \partial_{\xi} \Psi(\xi, x, y)\right|-f\left(|\xi|^{2}\right)\right\} \phi(y) d_{1} y d_{1} \xi
\end{aligned}
$$

where $g \in C_{0}^{\infty}\left(\mathbf{R}^{n}\right)$ was defined in part (i) of this proof. The first integral operator is compact because the kernel is in $L^{2}\left(\mathbf{R}^{3 n}\right)$. Therefore to prove $J^{-} f\left(H_{0}\right)\left(J^{-}\right)^{*}-$ $f\left(H_{0}\right)$ is compact we need only show that the second integral expression defines a bounded linear operator whose norm goes to 0 with $\varepsilon$. Again we apply the Calderón-Vaillancourt theorem [2]. Since

$$
\begin{aligned}
& D_{x}^{\alpha} D_{y}^{\beta} D_{\xi}^{\gamma}\{1-g(\varepsilon x) g(\varepsilon y)\}\left\{f\left(|\Psi(\xi, x, y)|^{2}\right)\left|\operatorname{det} \partial_{\xi} \Psi(\xi, x, y)\right|-f\left(|\xi|^{2}\right)\right\} \\
&<C_{\alpha, \beta, \gamma}\left(1+\varepsilon^{-2}\right)^{-\varepsilon_{1 / 4}}
\end{aligned}
$$

$\left(|\alpha|,|\beta|,|\gamma|<\frac{1}{2} m_{0}\right)$, the result follows. 
Similar methods are applied in the next result to study $\left(J^{ \pm}\right)^{*} J^{ \pm}-1$. Because of the asymmetry of the dependence of $\theta^{ \pm}(x, \xi)$ on $x$ and $\xi$ (see (5.6)), $\left(J^{ \pm}\right)^{*} J^{ \pm}$is less tractable than $J^{ \pm}\left(J^{ \pm}\right)^{*}$.

LEMMA 5.4. For any $f \in C_{0}^{\infty}(\mathbf{R}), f\left(H_{0}\right)\left[\left(J^{ \pm}\right)^{*} J^{ \pm}-1\right] f\left(H_{0}\right)$ are compact operators. In particular $J^{ \pm}$satisfy $(\mathrm{H} 4)$.

ProOF. The second conclusion is a consequence of the first because the first conclusion implies that $R_{\sigma}^{ \pm}(f)\left[\left(J^{ \pm}\right)^{*} J^{ \pm}-1\right] R_{\sigma}^{ \pm}(f)$ are compact and because $e^{-i t H_{0}} \rightarrow$ 0 weakly as $|t| \rightarrow \infty$ by the Riemann Lebesgue lemma. These two observations imply

$$
R^{ \pm}(f)\left[\left(J^{ \pm}\right)^{*} J^{ \pm}-1\right] R^{ \pm}(f) e^{-i t H_{0}} \rightarrow 0 \text { strongly as }|t| \rightarrow \infty
$$

which is (H4).

Clearly, proving the first statement is equivalent to showing

$$
\mathcal{f}\left(H_{0}\right)\left[\left(J^{ \pm}\right)^{*} J^{ \pm}-1\right] f\left(H_{0}\right) \digamma^{*}
$$

are compact. ( $₹$ is the Fourier transform.) We consider the "-" case, the "+" case being similar (or see (5.8)). The argument parallels that for Lemma 5.3, part (i). If $\psi \in S\left(\mathbf{R}^{n}\right)$ and $\psi(\xi)=0$ when $|\xi|<d$ for some $d>0$, then by (5.9)

$$
\begin{aligned}
\mathcal{F} f\left(H_{0}\right)\left(J^{-}\right)^{*} J^{-} f\left(H_{0}\right) \mathcal{F}^{*} \psi(\zeta)=f & \left(\frac{1}{2}|\zeta|^{2}\right) \int_{\mathbf{R}^{n}} \int_{\mathbf{R}^{n}} e^{i x \cdot(\xi-\zeta)} \\
& \cdot e^{i\left(\theta^{-}(x, \xi)-\theta^{-}(x, \zeta)\right)} f\left(\frac{1}{2}|\xi|^{2}\right) \psi(\xi) d_{1} \xi d_{1} x .
\end{aligned}
$$

Since

$$
\theta^{-}(x, \xi)-\theta^{-}(x, \varsigma)=\left(\int_{0}^{1} \nabla_{\xi} \theta^{-}(x, \varsigma+s(\xi-\varsigma)) d s\right) \cdot(\xi-\varsigma),
$$

the appropriate change of variable to reduce the above expression to a pseudodifferential operator is

$$
x^{\prime}=\Phi(x, \xi, \varsigma)=\int_{0}^{1} \nabla_{\xi} \theta^{-}(x, \zeta+s(\xi-\varsigma)) d s+x .
$$

From (5.6) we have

$$
\left|D_{x}^{\alpha} D_{\xi}^{\beta} D_{\zeta}^{\gamma}\{\Phi(x, \xi, \varsigma)-x\}\right|<C \eta\langle x\rangle^{1-|\alpha|-\varepsilon_{1}}\left(1+|\xi|^{2}+|\zeta|^{2}\right)^{-1 / 4}
$$

$\left(|\alpha|,|\beta+\gamma|<m_{0}\right)$ and we see that the function $x \rightarrow \Phi(x, \xi, \zeta)$ has an inverse $\Psi\left(x^{\prime}, \xi, \zeta\right)$ on all of $\mathbf{R}^{n}$ for each fixed $\xi$ and $\zeta$ provided $\eta$ is chosen suitably small. Moreover $\Psi \in C^{\infty}\left(\mathbf{R}^{3 n}\right)$ and

$$
\begin{array}{r}
\left|D_{x^{\prime}}^{\alpha} D_{\xi}^{\beta} D_{\zeta}^{\gamma}\left(\partial_{x^{\prime}} \Psi\left(x^{\prime}, \xi, \zeta\right)-1\right)\right|<C_{\alpha, \beta, \gamma}\left\langle x^{\prime}\right\rangle^{-|\alpha|-\varepsilon_{1}}\left(1+|\xi|^{2}+|\zeta|^{2}\right)^{-1 / 4} \\
\left(|\alpha|,|\beta|,|\gamma|<m_{0} / 2\right)
\end{array}
$$


To avoid integrability problems introduce a cut-off function. Choose $g \in C_{0}^{\infty}\left(\mathbf{R}^{n}\right)$ so that $g(x)=1$ if $|x|<1$ and $g(x)=0$ if $|x|>2$. Then

$$
\begin{gathered}
\mathcal{F}\left(H_{0}\right)\left(J^{-}\right)^{*} J^{-} f\left(H_{0}\right) \mathcal{F}^{*} \psi(\varsigma) \\
=\lim _{\varepsilon \rightarrow 0+} f\left(\frac{1}{2}|\zeta|^{2}\right) \int_{\mathbf{R}^{n}} \int_{\mathbf{R}^{n}} e^{i(\xi-\varsigma) \cdot \Phi(x, \xi, \varsigma)} \\
\cdot g(\varepsilon \Phi(x, \xi, \zeta)) f\left(\frac{1}{2}|\xi|^{2}\right) \psi(\xi) d_{1} \xi d_{1} x^{\prime} \\
=\lim _{\varepsilon \rightarrow 0+} f\left(\frac{1}{2}|\zeta|^{2}\right) \int_{\mathbf{R}^{n}} \int_{\mathbf{R}^{n}} e^{i x^{\prime} \cdot(\xi-\varsigma)} g\left(\varepsilon x^{\prime}\right) \\
\cdot f\left(\frac{1}{2}|\xi|^{2}\right)\left|\operatorname{det} \partial_{x^{\prime}} \Psi\left(x^{\prime}, \xi, \varsigma\right)\right| \psi(\xi) d_{1} \xi d_{1} x^{\prime}
\end{gathered}
$$

by changing variables. Applying the Fourier inversion formula to $\left[f\left(H_{0}\right)\right]^{2}$, we have

$$
\begin{aligned}
\mathcal{F} f\left(H_{0}\right)\left[\left(J^{-}\right)^{*} J^{-}-1\right] & f\left(H_{0}\right) \mathcal{F}^{*} \psi(\varsigma) \\
=\lim _{\varepsilon \rightarrow 0+} f\left(\frac{1}{2}|\zeta|^{2}\right) & \int_{\mathbf{R}^{n}} \int_{\mathbf{R}^{n}} e^{i x^{\prime} \cdot(\xi-\varsigma)} g\left(\varepsilon x^{\prime}\right) \\
& \cdot f\left(\frac{1}{2}|\xi|^{2}\right)\left\{\left|\operatorname{det} \partial_{x^{\prime}} \Psi\left(x^{\prime}, \xi, \varsigma\right)\right|-1\right\} \psi(\xi) d_{1} \xi d_{1} x .
\end{aligned}
$$

For fixed $\varepsilon$, the above integral defines a compact integral operator because the kernel is in $L^{2}\left(\mathbf{R}^{3 n}\right)$. Therefore to prove compactness it suffices to show that these operators converge in operator norm. This is done by appealing to the CalderónVaillancourt theorem [2]. By (5.10), we have: for $0<\varepsilon_{1}<\varepsilon_{0}$

$$
\begin{gathered}
\left|D_{x^{\prime}}^{\alpha} D_{\xi}^{\beta} D_{\zeta}^{\gamma}\left\{\left(g\left(\varepsilon^{\prime} x\right)-g(\varepsilon x)\right) f\left(\frac{1}{2}|\zeta|^{2}\right) f\left(\frac{1}{2}|\xi|^{2}\right)\left(\operatorname{det}\left(\partial_{x} \Psi(x, \xi, \varsigma)\right)-1\right)\right\}\right| \\
<C_{\alpha, \beta, \gamma}\left(1+(1 / \varepsilon)^{2}\right)^{\varepsilon_{1 / 4}}
\end{gathered}
$$

for all multi-indices $\alpha, \beta, \gamma$, so that $|\alpha|,|\beta|,|\gamma|<m_{0} / 2$. Therefore, by the CalderónVaillancourt theorem, $f f\left(H_{0}\right)\left[\left(J^{-}\right)^{*} J^{-}-1\right] f\left(H_{0}\right) \mathcal{F}^{*}$ is the limit of a sequence of compact operators which is Cauchy in operator norm. This establishes the lemma.

6. Long range nonoscillatory potentials. In this section we prove (Theorem 6.1) the special case of Theorem 1.1 when $U=0$. Thus, we consider $H_{0}=-\frac{1}{2} \Delta$ and $H=H_{0}+V_{s}+V$ where $V_{s}$ satisfies (SR) ${ }_{1}$ and $V$ satisfies (LR1). (See the Introduction.)

THEOREM 6.1. Let $V_{s}$ satisfy (SR) ${ }_{1}$ and $V$ satisfy (LR1), and let $J^{ \pm}$be the bounded operators on $L^{2}\left(\mathbf{R}^{n}\right)$ defined by (5.1). Let $H_{0}=-\frac{1}{2} \Delta$ and $H=H_{0}+$ $V_{s}+V$. Then $H$ is selfadjoint with domain $D(H)=D\left(H_{0}\right)$. The wave operators $\Omega^{ \pm} \equiv \Omega^{ \pm}\left(H, H_{0} ; J^{ \pm}\right)$exist and are complete (i.e. their ranges $R\left(\Omega^{ \pm}\right)$are both equal to the subspace of continuity of $H)$, and $\left(\Omega^{ \pm}-J^{ \pm}\right) R_{\sigma}^{ \pm}(f)$ are compact for any $f \in C_{0}^{\infty}((0, \infty))$ and $\sigma, \frac{1}{2} \sigma_{1}<\sigma<1\left(\sigma_{1}\right.$ as in (5.4)). In particular $H$ has no singularly continuous spectrum.

REMARK 6.2. (a) The proof that $H$ is selfadjoint is elementary; it goes as follows. First $V_{s}+V$ is $H_{0}$-compact, i.e. $\left(V_{s}+V\right)\left(H_{0}+1\right)^{-1}$ is compact because 
$V_{s}$ is by hypothesis and $V\left(H_{0}+1\right)^{-1}$ is the limit in operator norm of the operators $F(|x|<r) V\left(H_{0}+1\right)^{-1} E_{0}([0, r))$ as $r \rightarrow \infty$, which are compact (by Theorem VI.23, p. 210 of $[40])$. Consequently $H$ is selfadjoint on $D(H)=D\left(H_{0}\right)$ by the KatoRellich theorem $([41$, p. 162] or see $[43$, Corollary 2, p. 113]). Also the essential spectrum of $H$ is the same as that of $H_{0}$, i.e. $[0, \infty)$ [43, Corollary 2, p. 113]. Therefore $E_{c} E([0, \infty))=E_{c}$. Theorem 6.1 can be established therefore by verifying hypotheses (H1) through (H6) and appealing to Theorem 3.1 and Corollary 4.4.

(b) The assumption (SR) 1 in Theorem 6.1 is convenient but it is not the best possible. We require the following conditions for the following proofs to apply with no substantial change:

(1) $H=-\Delta+V+V_{s}$ is selfadjoint,

(2) $(H-z)^{-1}-\left(H_{0}-z\right)^{-1}$ is compact for some (hence all) $z \in \mathbf{C} \backslash \mathbf{R}$,

(3) $\left\|\left(H_{0}+1\right)^{-1} g_{1}(x / r) V_{s}(H-z)^{-1}\right\| \in L^{1}((1, \infty) d r)$, for some $k \in \mathbf{N}$ and $z \in \mathbf{C} \backslash \mathbf{R}$, with $g_{1}$ as in (SR) ${ }_{1}$.

We must therefore verify hypotheses (H1) through (H6). Hypothesis (H1) is verified with $\mathcal{H}=L^{2}\left(\mathbf{R}^{n}\right)$ because $H$ is selfadjoint (Remark $6.2(\mathrm{a})$ ) and $J^{ \pm}$are bounded (Lemma 5.3, part (ii)). We proceed to check the remaining five hypotheses in a series of lemmas. To check (H2) and (H3), we require two lemmas. To state the first, introduce the following functions. For each $\sigma, \sigma^{\prime},-1<\sigma^{\prime}<\sigma<1$, let $\chi_{\sigma^{\prime}, \sigma}$ be a real $C^{\infty}([-1,1])$ function so that

$$
\chi_{\sigma^{\prime}, \sigma}(r)= \begin{cases}1 & \text { if } r>\frac{1}{3}\left(2 \sigma+\sigma^{\prime}\right), \\ 0 & \text { if } r<\frac{1}{3}\left(\sigma+2 \sigma^{\prime}\right) .\end{cases}
$$

LEMMA 6.3. Suppose $q_{1} \in C^{\infty}\left(\mathbf{R}^{2 n}\right)$ and that for any compact set $K \subseteq \mathbf{R}^{n}\{0\}$ and all $\beta$ in $\mathrm{N}^{n}$

$$
\left|D_{\xi}^{\beta} q_{1}(x, \xi)\right|<C_{\beta, K}\langle x\rangle^{|\beta|\left(1-\varepsilon_{0}\right)} \quad \text { for all } x \in \mathbf{R}^{n}, \xi \in K\left(\varepsilon_{0}\right. \text { as in (LR1)). }
$$

Suppose also that $q_{1}(x, \xi)=0$ if $|x|<1$. For each $\sigma, \sigma^{\prime},-1<\sigma^{\prime}<\sigma<1$, define the operators $Q^{ \pm}$on $\left.\mathcal{F}^{*}\left(C_{0}^{\infty}\left(\mathbf{R}^{n}\right) \backslash\{0\}\right)\right)$ by

$$
Q^{ \pm} \phi(x)=\int_{\mathbf{R}^{n}} e^{i x \cdot \xi} q_{1}(x, \xi)\left\{1-\chi_{\sigma^{\prime}, \sigma}\left(\mp \frac{x \cdot \xi}{|x||\xi|}\right)\right\} \hat{\phi}(\xi) d \xi
$$

$\left(\phi \in C_{0}^{\infty}\left(\mathbf{R}^{n} \backslash\{0\}\right)\right)$. Then, for each $f \in C_{0}^{\infty}((0, \infty)), Q^{ \pm} R_{0}^{ \pm}(f)$ (which is defined on $S\left(\mathbf{R}^{n}\right)$ ) extends to a compact operator on $L^{2}\left(\mathbf{R}^{n}\right)$, provided $\delta$ in (2.2) is small enough $\left(\delta=\delta\left(\sigma-\sigma^{\prime}, f\right)\right)$. Moreover, for each $l \in \mathbf{N}$

$$
\left\|F(|x|>r) Q^{ \pm} R_{\sigma}^{ \pm}(f)\right\|<C_{l}(1+r)^{-l}, \quad r>0 .
$$

Essentially this result says that $R_{\sigma}^{ \pm}(f) \phi$ is localized, as would be expected, on the support $\chi_{\sigma^{\prime}, \sigma}(\mp x \cdot \xi /|x||\xi|)$. A proof of Lemma 6.3 is provided in the Appendix. Observe that, if $q_{1}(x, \xi)=e^{i \theta^{ \pm}(x, \xi)}$, then $q_{1}$ satisfies (6.2) (by (5.7)).

LEMMA 6.4. Suppose $q_{2} \in C^{\infty}\left(\mathbf{R}^{2 n}\right)$ and for every compact set $K \subseteq \mathbf{R}^{n} \backslash\{0\}$ and every $\beta \in \mathbf{N}^{n}$

$$
\left|D_{\xi}^{\beta} q_{2}(x, \xi)\right|<C_{\beta, K}\langle x\rangle^{|\beta|\left(1-\varepsilon_{0}\right)} \quad \text { for all }(x, \xi) \in \mathbf{R}^{n} \times K
$$

$\left(\varepsilon_{0}\right.$ as in (LR1)). Define the operator $Q$ on $\mathcal{F}^{*}\left(C_{0}^{\infty}\left(\mathbf{R}^{n} \backslash\{0\}\right)\right)$ by

$$
Q \phi(x)=\int_{\mathbf{R}^{n}} e^{i x \cdot \xi} q_{2}(x, \xi) \hat{\phi}(\xi) d \xi, \quad \hat{\phi} \in C_{0}^{\infty}\left(\mathbf{R}^{n} \backslash\{0\}\right)
$$


Then, for any $f \in C_{0}^{\infty}((0, \infty))$, any $\sigma,-1 \leq \sigma \leq 1$, and any $l \in \mathbf{N}$

$$
\left\|F\left(|x|<\frac{1}{3} r\right) Q R_{\sigma}^{ \pm}(f) F\left(\left|x^{\prime}\right|>r\right)\right\|<C_{l}(1+r)^{-l} .
$$

A proof of Lemma 6.4 is provided in the Appendix.

Verify now (H2) and (H3).

Lemma 6.5. Let $H, H_{0}$, and $J^{ \pm}$be as in Theorem 6.1. Then (H2) and (H3) are valid.

ProOF. We consider only the "-" case; the "+" case is similar. (Or use (5.8) and (2.4).)

We choose the constant $\sigma_{0}$ of (H2) and (H3) so that $\sigma_{1}<\sigma_{0}<0$, where $\sigma_{1}$ is the constant in the definition (5.4) of $\theta^{ \pm}$. Choose $g_{1} \in C^{\infty}\left(\mathbf{R}^{n}\right)$ satisfying (1.4). Let $f \in C_{0}^{\infty}((0, \infty))$. We shall prove that, for $z \in \mathbf{C} \backslash \mathbf{R}$, and $\sigma, \sigma_{0} \leq \sigma<1$,

$$
\left\|(H-z)^{-1}\left(H J^{-}-J^{-} H_{0}\right) R_{\sigma}^{-}(f) g_{1}\left(x^{\prime} / r\right)\right\| \in L^{1}((1, \infty), d r) .
$$

Since the left side of (6.4) is a decreasing function of $r$, it must go to 0 as $r \rightarrow \infty$, by (6.3). Thus

$$
\begin{aligned}
& (H-z)^{-1}\left(H J^{-}-J^{-} H_{0}\right) R_{\sigma}^{-}(f) \\
& \quad=\lim _{r \rightarrow \infty}(H-z)^{-1}\left(H J^{-}-J^{-} H_{0}\right) R_{0}^{-}(f)\left(1-g\left(\frac{x^{\prime}}{r}\right)\right) .
\end{aligned}
$$

Thus $(H-z)^{-1}\left(H J-J^{-} H_{0}\right) R_{\sigma}^{-}(f)$ is the limit of compact operators and hence compact $\left(f\left(H_{0}\right)\left(1-g_{1}\left(x^{\prime} / r\right)\right)\right.$ is clearly compact). Therefore (6.4) implies (H2) and it clearly implies (H3).

Thus it suffices to prove (6.4). As a preliminary we show that $\left(H_{0}+1\right)^{k} J^{-} f\left(H_{0}\right)$ is bounded for any $k \in \mathbf{N}$. This is an immediate consequence of Lemma 5.3, part (i) applied when $q$ there is

$$
q(x, \xi)=f\left(\frac{1}{2}|\xi|^{2}\right) e^{-i x \cdot \xi-i \theta^{-}(x, \xi)}\left(1-\Delta_{x}\right)^{k}\left\{e^{i x \cdot \xi+i \theta^{-}(x, \xi)}\right\}
$$

which is bounded along with all its derivatives by (5.7). Now consider the left-hand side of (6.4); it is bounded by

$$
\begin{aligned}
& \left\|V_{s}(H-z)^{-1}\right\|\left\|\left(1-g_{1}\left(\frac{6 x}{r}\right)\right) J^{-} R_{\sigma}^{-}(f) g_{1}\left(\frac{x^{\prime}}{r}\right)\right\| \\
& \quad+\left\|\left(H_{0}+1\right)(H-\bar{z})^{-1}\right\|\left\|\left(H_{0}+1\right)^{-1} V_{s} q_{1}\left(\frac{6 x}{r}\right)\left(H_{0}+1\right)^{-k}\right\| \\
& \quad \cdot\left\|\left(H_{0}+1\right)^{k} J^{-} R_{\sigma}^{-}(f)\right\|+\left\|(H-z)^{-1}\left[\left(H_{0}+V\right) J^{-}-J^{-} H_{0}\right] R_{\sigma}^{-}(f) g_{1}\left(\frac{x^{\prime}}{r}\right)\right\| .
\end{aligned}
$$

(Recall that $(H-z)^{-1}\left(H J^{-}-J^{-} H_{0}\right) R_{\sigma}^{-}(f)$ is interpreted as a sesquilinear form.) The first term is integrable by Lemma 6.4 and so is the second by assumption (SR $)_{1}$. $\left(\left(H_{0}+1\right)(H-z)^{-1}\right.$ is bounded because $D(H)=D\left(H_{0}\right)$.) Thus to prove (6.4), we need only consider the third term or equivalently prove (6.4) in the case $V_{s}=0$. Assume therefore that $V_{s}=0$.

If $\phi \in S\left(\mathbf{R}^{n}\right)$ and $\phi \in C_{0}^{\infty}\left(\mathbf{R}^{n} \backslash\{0\}\right)$ so that $J^{-} \phi \in S\left(\mathbf{R}^{n}\right)$ (by (5.9)), then we may write

$$
\left(H J^{-}-J^{-} H_{0}\right) \phi(x)=\int_{\mathbf{R}^{n}} e^{i x \cdot \xi} q_{2}(x, \xi) \phi(\xi) d \xi
$$


where

$$
q_{2}(x, \xi)=e^{i \theta^{-}(x, \xi)}\left\{\frac{1}{2}\left|\xi+\nabla_{x} \theta^{-}(x, \xi)\right|^{2}-\frac{1}{2}|\xi|^{2}+V(x)-\frac{i}{2} \Delta_{x} \theta^{-}(x, \xi)\right\} .
$$

Using (5.7), one easily checks that $q_{2}$ satisfies the hypotheses of Lemma 6.4. We shall apply Lemma 6.4 in a moment but first observe that, because the left side of (6.4) is bounded and decreasing in $r$, we may restrict attention to when $r$ is large. The left side of (6.4) is bounded by

$$
\begin{array}{r}
\left\|(H-z)^{-1}\right\|\left\|\left(1-g_{1}(6 x / r)\right)\left(H J^{-}-J^{-} H_{0}\right) R_{\sigma}^{-}(f) g_{1}(x / r)\right\| \\
+\left\|(H-z)^{-1} g_{1}(6 x / r)\left(H J^{-}-J^{-} H_{0}\right) R_{\sigma}^{-}(f) g_{1}(x / r)\right\| .
\end{array}
$$

By Lemma 6.4, the first term is an integrable function of $r$ and we are left to consider the second term. For $\phi \in S\left(\mathbf{R}^{n}\right)$

$$
\begin{aligned}
g_{1}\left(\frac{6 r}{r}\right) & \left(H J^{-}-J^{-} H_{0}\right) R_{\sigma}^{-}(f) g_{1}\left(\frac{x}{r}\right) \phi(x) \\
= & \int_{\mathbf{R}^{n}} e^{i x \cdot \xi} q_{1}(x, \xi)\left(1-\chi_{\sigma_{1} \sigma_{0}}\left(\frac{x \cdot \xi}{|x||\xi|}\right)\right)\left(R_{\sigma}^{-}(f) g_{1}\left(\frac{x}{r}\right) \hat{\phi}\right) \widehat{(}(\xi) d \xi \\
& +\int_{\mathbf{R}^{n}} e^{i x \cdot \xi+i \theta^{-}(x, \xi)} q(x, \xi)\left(R_{\sigma}^{-}(f) g_{1}\left(\frac{x}{r}\right) \phi\right) \widehat{(\xi) d \xi}
\end{aligned}
$$

where

$$
\begin{gathered}
q_{1}(x, \xi)=g_{1}\left(\frac{6 x}{r}\right) q_{2}(x, \xi) \\
q(x, \xi)=g_{1}\left(\frac{6 x}{r}\right) e^{-i \theta^{-}(x, \xi)} q_{2}(x, \xi) \chi_{\sigma_{1} \sigma_{0}}\left(\frac{(x \mid \xi)}{|x||\xi|}\right) .
\end{gathered}
$$

We shall show that both terms on the right side of (6.5) are bounded by $C(1+r)^{-1-\varepsilon_{0}}\|\phi\|$ which will complete the proof. This is true for the first term by Lemma 6.3. As for the second term, we apply Lemma 5.3. To do so, we require estimates on the derivatives of $q$ which are obtained as follows. By Lemma 5.2

$$
q(x, \xi)=-\frac{i}{2} g_{1}\left(\frac{6 x}{r}\right) \Delta_{x} \theta^{-}(x, \xi) \chi_{\sigma_{1} \sigma_{0}}\left(\frac{x \cdot \xi}{|x||\xi|}\right)
$$

provided $\xi$ is in the support of $f\left(\frac{1}{2}|\xi|^{2}\right)$ and $r$ is suitably large. For such $\xi$ and $r$, we have by $(5.7)$

$$
\left|D_{x}^{\alpha} D_{x}^{\beta} q(x, \xi)\right|<C_{\alpha, \beta}(1+r)^{-1-\varepsilon_{0}}
$$

for all multi-indices $\alpha, \beta$. Applying Lemma 5.3 gives the result.

Proceed now to verify (H4), (H5) and (H6). Since (H4) was dealt with in Lemma 5.4, we consider (H5) next.

LEMMA 6.6. Let $H, H_{0}$ and $J^{ \pm}$be as in Theorem 6.1. Then (H5) is valid.

Proof. Choose $I \subseteq(0, \infty)$ to be compact and $\left\{f_{m}\right\}_{m \in N}$ in $C_{0}^{\infty}((0, \infty))$ so that $f_{m}(\lambda)=1$ if $1 / m<\lambda<m$, and $f_{m}$ is real. We must prove that the operators

$$
E_{c} E(I)\left[J^{ \pm} f_{m}\left(H_{0}\right)\left(J^{ \pm}\right)^{*}-1\right] E(I) E_{c}
$$

are compact for all $m$ suitably large. By Lemma 5.3, part (ii), $J^{ \pm} f_{m}\left(H_{0}\right)\left(J^{ \pm}\right)^{*}-$ $f_{m}\left(H_{0}\right)$ is compact and so it suffices to prove that

$$
=E_{c} E(I)\left[f_{m}\left(H_{0}\right)-1\right] E(I) E_{c}
$$


is compact. If $m$ is so large that $I \subseteq(1 / m, m)$, then $E(I)=f_{m}(H) E(I)$ and it is therefore enough to prove $f_{m}\left(H_{0}\right)-f_{m}(H)$ is compact. Consider therefore the set $A$ of all continuous functions $f$ such that $f(H)-f\left(H_{0}\right)$ is compact. We shall show, by using the Stone-Weierstrass theorem, that $A$ consists of all continuous functions $f$ for which $f(\lambda) \rightarrow 0$ as $|\lambda| \rightarrow \infty$. By the Stone-Weierstrass theorem, we need only show $A$ is a closed nonvoid algebra, closed under complex conjugation. Certainly $A$ is nonvoid because

$$
(H-z)^{-1}-\left(H_{0}-z\right)^{-1}=(H-z)^{-1}\left(V+V_{s}\right)\left(H_{0}-z\right)^{-1}
$$

is compact. (See Remark 6.2(a).) Also $A$ is clearly an algebra which is closed under complex conjugation. Finally $A$ is closed in the topology of uniform convergence (on the one-point compactification of $\mathbf{R}$ ), because the set of compact operators is closed in operator norm. Now, by the Stone-Weierstrass theorem $f_{m}(H)-f_{m}\left(H_{0}\right)$ is compact.

LEMMA 6.7. Under the assumptions of Theorem 6.1, (H6) is valid.

PROOF. We prove that $R_{\sigma}^{-}\left(f_{1}\right)\left(J^{+}\right)^{*} J^{-} R_{\sigma^{\prime}}^{+}\left(f_{2}\right)$ is compact whenever $\sigma, \sigma^{\prime}>0$ and $f_{1}, f_{2} \in C_{0}^{\infty}((0, \infty))$. The other case when all signs are reversed is proved similarly or by using (2.4) and (5.8).

Our task may be simplified by applying Lemma 6.3. To do so, define $g_{1} \in$ $C^{\infty}\left(\mathbf{R}^{n}\right)$ as before (in (1.4)). Then for any $\phi \in S\left(\mathbf{R}^{n}\right)$

$$
\begin{aligned}
J^{-} R_{\sigma^{\prime}}^{+}\left(f_{2}\right) \phi(x)= & \int_{\mathbf{R}^{n}} e^{i x \cdot \xi+i \theta^{-}(x, \xi)}\left(1-g_{1}(x)\right)\left(R_{\sigma^{\prime}}^{+}\left(f_{2}\right) \phi\right)^{\Upsilon}(\xi) d_{1} \xi \\
& +\int_{\mathbf{R}^{n}} e^{i x \cdot \xi+i \theta^{-}(x, \xi)} g_{1}(x)\left(1-\chi_{0, \sigma^{\prime}}\left(-\frac{x \cdot \xi}{|x||\xi|}\right)\right)\left(R_{\sigma^{\prime}}^{+}\left(f_{2}\right) \phi\right)^{\Upsilon}(\xi) d_{1} \xi \\
& +\int_{\mathbf{R}^{n}} e^{i x \cdot \xi+i \theta^{-}(x, \xi)} g_{1}(x) \chi_{0, \sigma^{\prime}}\left(-\frac{x \cdot \xi}{|x||\xi|}\right)\left(R_{\sigma^{\prime}}^{+}\left(f_{2}\right) \phi\right)^{-}(\xi) d_{1} \xi .
\end{aligned}
$$

The first two integral expressions define compact operators; the first has a square integrable kernel and the second is by Lemma 6.3. A similar decomposition applies to $J^{+} R_{\sigma}^{-}\left(\bar{f}_{1}\right)$. Therefore to prove the compactness of $R_{\sigma}^{-}\left(f_{1}\right)\left(J^{+}\right)^{*} J^{-} R_{\sigma^{\prime}}^{+}\left(f_{2}\right)$, it suffices to prove the compactness of the integral operator which maps $\phi \in S\left(\mathbf{R}^{n}\right)$ to

$$
\begin{aligned}
\int_{\mathbf{R}^{n}} & \int_{\mathbf{R}^{n}} e^{i x \cdot(\xi-\varsigma)} e^{i\left(\theta^{-}(x, \xi)-\theta^{+}(x, \zeta)\right)} \\
\cdot & \left(g_{1}(x)\right)^{2} \chi_{0, \sigma^{\prime}}\left(-\frac{x \cdot \xi}{|x||\xi|}\right) \chi_{0, \sigma}\left(\frac{x \cdot \zeta}{|x||\zeta|}\right) \\
& \cdot f_{1}\left(\frac{1}{2}|\zeta|^{2}\right) f_{2}\left(\frac{1}{2}|\xi|^{2}\right) \hat{\phi}(\xi) d_{1} \xi d_{1} x
\end{aligned}
$$

(The inner integral over $\xi$ defines a function of $x$ which is in $S\left(\mathbf{R}^{n}\right)$; see the proof of (5.9).) To complete the proof we shall write $e^{i x \cdot(\xi-\varsigma)}=|\xi-\rho|^{-2 N}\left(-\Delta_{x}\right)^{N} e^{i x \cdot(\xi-\varsigma)}$ (for any integer $N$ ) and integrate by parts in the above integral. For this to make sense we must check that $|\varsigma-\xi| \neq 0$, but

$$
|\varsigma-\xi| \geq \frac{1}{|x|}[x \cdot \varsigma-x \cdot \xi]>\frac{1}{3} \sigma|\varsigma|+\frac{1}{3} \sigma^{\prime}|\xi|
$$


on the support of the integrand in (6.6). Technically, to justify the integration by parts, we introduce a cut-off function $g$ where $g(x)=1-g_{1}(x)$. Then (6.6) may be rewritten as

$$
\begin{aligned}
& \lim _{r \rightarrow \infty} \int_{\mathbf{R}^{n}} \int_{\mathbf{R}^{n}} e^{i x \cdot(\xi-\varsigma)} e^{i\left(\theta^{-}(x, \xi)-\theta^{+}(x, \zeta)\right)} g\left(\frac{x}{r}\right)\left(g_{1}(x)\right)^{2} \\
& \cdot \chi_{0, \sigma^{\prime}}\left(-\frac{x \cdot \xi}{|x||\zeta|}\right) \chi_{0, \sigma}\left(\frac{x \cdot \zeta}{|x||\zeta|}\right) f_{1}\left(\frac{1}{2}|\zeta|^{2}\right) f_{2}\left(\frac{1}{2}|\xi|^{2}\right) \hat{\phi}(\xi) d_{1} \xi d_{1} x \\
&= \lim _{r \rightarrow \infty} \int_{\mathbf{R}^{n}} \int_{\mathbf{R}^{n}} e^{i x(\xi-\zeta)}\left(-\Delta_{x}\right)^{N} \\
& \cdot\left\{e^{i\left(\theta^{-}(x, \zeta)-\theta^{+}(x, \zeta)\right)} g\left(\frac{x}{r}\right)\left(g_{1}(x)\right)^{2} \cdot \chi_{0, \sigma^{\prime}}\left(-\frac{x \cdot \xi}{|x||\xi|}\right) \chi_{0, \sigma}\left(\frac{x \cdot \zeta}{|x||\zeta|}\right)\right\} \\
& \cdot|\xi-\zeta|^{-2 N} f_{1}\left(\frac{1}{2}|\zeta|^{2}\right) f_{2}\left(\frac{1}{2}|\xi|^{2}\right) \hat{\phi}(\xi) d_{1} x d_{1} \xi
\end{aligned}
$$

To estimate the integrand, recall (5.7):

$$
\begin{gathered}
\left|\left(-\Delta_{x}\right)^{N}\left\{e^{i\left(\theta^{-}(x, \xi)-\theta^{+}(x, \zeta)\right)} g\left(\frac{x}{r}\right)\left(g_{1}(x)\right)^{2} \chi_{0, \sigma^{\prime}}\left(-\frac{x \cdot \xi}{|x||\xi|}\right) \chi_{0, \sigma}\left(\frac{x \cdot \zeta}{|x||\zeta|}\right)\right\}\right| \\
<C_{N}\langle x\rangle^{-N \varepsilon_{0}}
\end{gathered}
$$

(assuming for the moment that $\varepsilon_{0} \leq 1$ ). Therefore, if $N$ is so large that $N \varepsilon_{0}>\frac{1}{2} n$, then the integrand in the last integral expression above is in $L^{2}\left(\mathbf{R}^{3 n}\right)$. We may therefore take the limit inside the integrals (eliminating $g$ ) and we are left with an integral operator with kernel in $L^{2}\left(\mathbf{R}^{3 n}\right)$ which is therefore compact. The proof is complete.

PROOF OF THEOREM 6.1. As noted in Remark 6.2(a) the theorem follows once hypotheses (H1) through (H6) have been verified. Since $H$ is selfadjoint (Remark $6.2(\mathrm{a})$ ) and $J^{ \pm}$are bounded by Lemma 5.3, part (ii), hypothesis (H1) is verified. We have verified (H2) and (H3) in Lemma 6.5, (H4) in Lemma 5.4, (H5) in Lemma 6.6 and (H6) in Lemma 6.7.

7. Theorem 1.1. In this section, we prove Theorem 1.1. The Hamiltonian is

$$
H=-\frac{1}{2} \Delta+\frac{1}{2}(\Delta U)+\frac{1}{2}|\nabla U|^{2}+V+V_{s}
$$

at least formally, but to avoid unnecessarily restrictive assumptions on $U, H$ will be defined as by means of sesquilinear forms. To be more precise we follow Combescure [3] (or [34]) and define the form

$$
H_{1}=\frac{1}{2} e^{-U}(\nabla / i) e^{2 U}(\nabla / i) e^{-U}
$$

(written in operator notation) with form domain

$$
D\left(H_{1}^{1 / 2}\right)=e^{U} D\left(\left(H_{0}+1\right)^{1 / 2}\right) \supseteq D\left(H_{1}\right) .
$$

(Recall that $U \in L^{\infty}\left(\mathbf{R}^{n}\right)$ by assumption (LR2) so that the multiplication operator $e^{U}$ is bounded and has bounded inverse $e^{-U}$.) This form is a densely defined, nonnegative selfadjoint operator $H_{1}[24$, Theorem 2.23, p. 331]. Now $H$ can be defined as $H=H_{1}+V+V_{s}$. To check that $H$ is well defined, observe that $\left(V+V_{s}\right)\left(H_{1}+1\right)^{-1}$ is compact because

$$
\left(V+V_{s}\right)\left(H_{1}+1\right)^{-1}=\left(V+V_{s}\right) e^{U}\left(H_{0}+1\right)^{-1 / 2}\left(H_{0}+1\right)^{1 / 2} e^{-U}\left(H_{1}+1\right)^{-1}
$$


and $V_{s} e^{U}\left(H_{0}+1\right)^{-1 / 2}$ is compact (see Remark 6.2(a)). It follows as in Remark 6.2(a) that $H$ is a selfadjoint operator with domain

$$
D(H)=D\left(H_{1}\right) \subseteq e^{U} D\left(\left(H_{0}+1\right)^{1 / 2}\right)
$$

and essential spectrum the same as that of $H_{1}$ which is a subset of $[0, \infty)$, because $H_{1}$ is nonnegative. Therefore

$$
E([0, \infty)) E_{c}=E_{c} .
$$

In addition, since $D(H)=D\left(H_{1}\right)$

$$
V_{s}(H-z)^{-1} \text { is compact for any } z \in \mathbf{C} \backslash \mathbf{R} \text {. }
$$

REMARK 7.1. The remainder of this section is devoted to verifying hypotheses (H1) through $(\mathrm{H} 6)$ for an appropriate choice of the operators $J^{ \pm}$. By Corollary 4.4, we will conclude the $\Omega^{ \pm} \equiv \Omega^{ \pm}\left(H, H_{0} ; J^{ \pm}\right)$exist and are isometries with range $E([0, \infty)) E_{c}\left(L^{2}\left(\mathbf{R}^{n}\right)\right)=E_{c}\left(L^{2}\left(\mathbf{R}^{n}\right)\right)$ (by $\left.(7.2)\right)$. This implies that the continuous spectrum of $H$ is $[0, \infty)$, by Lemma 3.2. The essential spectrum contains the continuous spectrum, i.e. $[0, \infty)$, but is also contained in $[0, \infty)$ according to the preceding discussion. Thus we will conclude that the essential spectrum is $[0, \infty)$. To verify Theorem 1.1 , we therefore only need to verify hypotheses (H1) through (H6). Moreover, we will also be able to conclude from Theorem 3.1 that $\left(\Omega^{ \pm}-J^{ \pm}\right) R_{\sigma}^{ \pm}(f)$ are compact for any $f \in C_{0}^{\infty}((0, \infty))$ and any $\sigma \geq \sigma_{0}$ (where $\sigma_{0}>\sigma_{1}$ and $\sigma_{1}$ is as in the definition (5.4) of $\theta^{ \pm}$).

It remains therefore to define $J^{ \pm}$and verify (H1) through (H6). The definition of $J^{ \pm}$is not quite the same as for the proof of Theorem 6.1 , where $J^{ \pm}$were defined by (5.1), but those operators again play a role and are therefore denoted by $J_{0}^{ \pm}$:

$$
J_{0}^{ \pm} \phi(x)=\int_{\mathbf{R}^{n}} e^{i x \cdot \xi+i \theta^{ \pm}(x, \xi)} \hat{\phi}(\xi) d_{1} \xi \quad\left(\phi \in S\left(\mathbf{R}^{n}\right)\right)
$$

(where $\theta^{ \pm}$are defined by (5.4)). We have already seen that $J_{0}^{ \pm}$extend to bounded operators on $L^{2}\left(\mathbf{R}^{n}\right)$ in Lemma 5.3 , part (ii). We define $J^{ \pm}=e^{U} J_{0}^{ \pm}$, where $e^{U}$ acts multiplicatively, and we have $J^{ \pm}$are bounded because $U \in L^{\infty}\left(\mathbf{R}^{n}\right)$ by assumption (LR2). Hypothesis (H1) is therefore satisfied. We proceed now to verify (H2) through (H6) in two lemmas. Before doing so it is instructive to do the formal computation analogous to (5.2) when $H=-\frac{1}{2} \Delta+\frac{1}{2} \Delta U+\frac{1}{2}|\nabla U|^{2}+V+V_{s}$ :

$$
\begin{aligned}
\left(H J^{-}\right. & \left.-J^{-} H_{0}\right) R_{\sigma}^{-}(f) \phi \\
= & V_{s} J^{-} R_{\sigma}^{-}(f) \phi+\int_{\mathbf{R}^{n}} e^{i x \cdot \xi+i\left(\theta^{-}(x, \xi)-i U(x)\right)} \\
& \cdot\left\{\frac{1}{2}\left|\xi+\nabla_{x} \theta^{-}(x, \xi)\right|^{2}-\frac{1}{2}|\xi|^{2}+V(x)-\frac{i}{2} \Delta_{x} \theta^{-}(x, \xi)\right. \\
& \left.+\left(\frac{1}{i} \nabla_{x}\right) \cdot U\left(\xi+\nabla_{x} \theta^{-}(x, \xi)\right)-U(x)\left(\frac{1}{i} \nabla_{x}\right) \cdot\left(\nabla_{x} \theta^{-}(x, \xi)\right)\right\} \\
& \cdot\left(R_{\sigma}^{-}(f) \phi\right)^{-}(\xi) d_{1} \xi,
\end{aligned}
$$

where we have written $\left(\nabla_{x} U\right)=\left(\nabla_{x}\right) U-U\left(\nabla_{x}\right)$. It is clear that the integrand is short range in $x$, but because $U$ may not be differentiable, the proof of (H2) will have to take a different approach. 
LEMMA 7.2. Let $H_{0}=-\frac{1}{2} \Delta$ and $H$ and $J^{ \pm}$be defined as above (i.e. as in Theorem 1.1). Then (H2) and (H3) are valid.

PrOOF. We prove only the "-" case of (H2) and (H3). The "+" case is similar (or use (5.8) and (2.4)).

This proof parallels that of Lemma 6.5 and so we shall only emphasize the additional arguments required. As in the proof of Lemma 6.5 (see (6.3)), to verify (H2) and (H3) it suffices to prove that

$$
\left\|(H-z)^{-1}\left(H J^{-}-J^{-} H_{0}\right) R_{\sigma}^{-}(f) g_{1}\left(x^{\prime} / r\right)\right\| \in L^{1}((1, \infty) d r)
$$

for $z \in \mathbf{C} \backslash \mathbf{R}, \sigma_{0} \leq \sigma<1$ and $f \in C_{0}^{\infty}((0, \infty))$, where $g_{1} \in C^{\infty}\left(\mathbf{R}^{n}\right)$ is as before $(\operatorname{see}(1.4))$.

As before we observe that $\left(H_{0}+1\right)^{k} J_{0}^{-} f\left(H_{0}\right)$ is a bounded operator. It is therefore possible to write the operator in (7.5) as

$$
\begin{aligned}
(H- & z)^{-1}\left(H J^{-}-J^{-} H_{0}\right) R_{\sigma}^{-}(f) g_{1}\left(\frac{x^{\prime}}{r}\right) \\
= & (H-z)^{-1} e^{-U}\left[\left(H_{0}+V\right) J_{0}^{-}-J_{0}^{-} H_{0}\right] R_{\sigma}^{-}(f) g_{1}\left(\frac{x^{\prime}}{r}\right) \\
& +\left\{(H-z)^{-1}\left(e^{-U}-e^{U}\right)\right\}\left\{J_{0}^{-} H_{0} R_{\sigma}^{-}(f) g_{1}\left(\frac{x^{\prime}}{r}\right)\right\} \\
& +\left\{\frac{1}{2}(H-z)^{-1} e^{-U}\left(\frac{1}{i} \nabla\right) \cdot\left(e^{2 U}-1\right)\right\} \cdot\left\{\left(\frac{1}{i} \nabla\right) J_{0}^{-} R_{\sigma}^{-}(f) g_{1}\left(\frac{x^{\prime}}{r}\right)\right\} \\
& +\left\{(H-z)^{-1} V\left(e^{U}-e^{-U}\right)\right\}\left\{J_{0}^{-} R_{\sigma}^{-}(f) g_{1}\left(\frac{x^{\prime}}{r}\right)\right\} \\
& +\left\{(H-z)^{-1} V_{s}\left(e^{U}-1\right)\right\}\left\{J_{0}^{-} R_{\sigma}^{-}(f) g_{1}\left(\frac{x^{\prime}}{r}\right)\right\} \\
& +\left\{(H-z)^{-1} e^{-U}\left(H_{0}+1\right)^{1 / 2}\left(H_{0}+1\right)^{-1 / 2} e^{U} V_{s}\right\} \\
& +\left\{\left(H_{0}+1\right)^{-k}\left(H_{0}+1\right)^{k} J_{0}^{-} R_{\sigma}^{-}(f) g_{1}\left(\frac{x^{\prime}}{r}\right)\right\}
\end{aligned}
$$

We shall now apply Lemma 6.4 to show that each term but the first has norm integrable in $r>1$. The estimates for each term except the first are all alike. For example the norm of the last term is bounded by

$$
\begin{aligned}
& \left\|V_{s}(H-z)^{-1}\right\|\left\|\left(1-g_{1}\left(\frac{6 x}{r}\right)\right) J_{0}^{-} R_{\sigma}^{-}(f) g_{1}\left(\frac{x^{\prime}}{r}\right)\right\| \\
& \quad+\left\|\left(H_{0}+1\right)^{1 / 2} e^{-U}(H-z)^{-1}\right\| \\
& \quad\left\|\left(H_{0}+1\right)^{-1 / 2} e^{U} V_{s} g_{1}\left(\frac{6 x}{r}\right)\left(H_{0}+1\right)^{-k}\right\|\left\|\left(H_{0}+1\right)^{k} J_{0}^{-} R_{\sigma}^{-}(f)\right\| .
\end{aligned}
$$

Both of the terms in (7.7) are integrable; the first by Lemma 6.4 and the second by assumption $(\mathrm{SR})_{1 / 2}$. The second through fifth terms of (7.6) are handled similarly. Each is the product of two bounded factors in braces between which we insert $1=g_{1}(6 x / r)+\left(1-g_{1}(6 x / r)\right)$. The first factor times $g_{1}(6 x / r)$ on the right has norm integrable in $r>1$ by assumption (LR2). The second factor times $\left(1-g_{1}(6 x / r)\right)$ is integrable by Lemma 6.4 . 
Therefore to prove (7.5), we need only prove that the norm of the first term on the right of (7.6) has norm integrable in $r>1$. The argument given in the proof of Lemma 6.5 applies here. (The argument starting after we assume $V_{s}=0$ there.) This completes the proof of (7.5).

Lemma 7.3. Let $H_{0}, H$ and $J^{ \pm}$be as in Theorem 1.1. Then (H4), (H5) and (H6) are valid.

ProOF. Observe that $\left(e^{U}-1\right)\left(H_{0}+1\right)^{-1 / 2}$ is compact, because

$$
\left(e^{U}-1\right)\left(H_{0}+1\right)^{-1 / 2}=\lim _{r \rightarrow \infty} F(|x|<r)\left(e^{U}-1\right)\left(H_{0}+1\right)^{-1 / 2} E_{0}([0, r]) .
$$

Therefore, for any $z \in \mathbf{C} \backslash \mathbf{R}$

$$
\left(e^{U}-1\right)(H-z)^{-1}=e^{U}\left(e^{U}-1\right)\left(H_{0}+1\right)^{-1 / 2}\left(H_{0}+1\right)^{1 / 2} e^{-U}(H-z)^{-1}
$$

is compact (see (7.1)). Similarly, if $f \in C_{0}^{\infty}((0, \infty))$, then

$$
\left(e^{U}-1\right) J_{0}^{ \pm} f\left(H_{0}\right)=\left(e^{U}-1\right)\left(H_{0}+1\right)^{-1}\left(H_{0}+1\right) J_{0}^{ \pm} f\left(H_{0}\right)
$$

are compact because $\left(H_{0}+1\right) J_{0}^{ \pm} f\left(H_{0}\right)$ are bounded. (See the proof of Lemma 6.4.)

To prove (H4) it suffices to prove the compactness of the operators

$$
\begin{aligned}
& f\left(H_{0}\right)\left[\left(J^{ \pm}\right)^{*} J^{ \pm}-1\right] f\left(H_{0}\right) \\
& \quad=f\left(H_{0}\right)\left[\left(J_{0}^{ \pm}\right)^{*} J_{0}^{ \pm}-1\right] f\left(H_{0}\right)+f\left(H_{0}\right)\left[\left(J_{0}^{ \pm}\right)^{*}\left(e^{2 U}-1\right) J_{0}^{ \pm}-1\right] f\left(H_{0}\right) .
\end{aligned}
$$

(See the proof of Lemma 5.4.) But the first term is compact by Lemma 5.4 and the second by (7.9).

Similarly, to prove (H6), we write

$$
\begin{aligned}
R_{\sigma_{1}}^{\mp}\left(f_{1}\right)\left(J^{ \pm}\right)^{*} J^{\mp} R_{\sigma_{2}}^{ \pm}\left(f_{2}\right)= & R_{\sigma_{1}}^{\mp}\left(f_{1}\right)\left(J_{0}^{ \pm}\right)^{*}\left(J_{0}^{ \pm}\right) R_{\sigma_{2}}^{ \pm}\left(f_{2}\right) \\
& +R_{\sigma_{1}}^{\mp}\left(f_{1}\right)\left(J_{0}^{ \pm}\right)^{*}\left(e^{2 U}-1\right) J^{\mp} R_{\sigma_{2}}^{ \pm}\left(f_{2}\right) .
\end{aligned}
$$

The first term is compact by Lemma 6.7 and the second by (7.9).

It remains to prove (H5). We must prove the compactness of

$$
E_{c} E(I)\left[e^{U} J_{0}^{ \pm} f_{m}\left(H_{0}\right)\left(J_{0}^{ \pm}\right)^{*} e^{U}-1\right] E(I) E_{c}
$$

or equivalently of

$$
E_{c} E(I)\left[J_{0}^{ \pm} f_{m}\left(H_{0}\right)\left(J_{0}^{ \pm}\right)^{*}-1\right] E(I) E_{c}
$$

(by (7.8)) for $m$ large enough. The proof of Lemma 6.6 will apply if we show that $(H-z)^{-1}-\left(H_{0}-z\right)^{-1}$ is compact for $z \in \mathbf{C} \backslash \mathbf{R}$. By (7.8) this is equivalent to proving the compactness of

$$
\begin{aligned}
& (H-z)^{-1}\left[H e^{U}-e^{-U} H_{0}\right]\left(H_{0}-z\right)^{-1} \\
& \quad=\frac{1}{2}(H-z)^{-1}\left[e^{-U}\left(\frac{1}{i} \nabla\right)\left(e^{2 u}-1\right)\left(\frac{1}{i} \nabla\right)+\left(V+V_{s}\right) e^{U}\right]\left(H_{0}-z\right)^{-1}
\end{aligned}
$$

and this last expression is compact by (7.3). The proof of Lemma 6.6 now applies to prove (H5).

PROOF OF THEOREM 1.1. It has already been noted in Remark 7.1 that the theorem follows once (H1) through (H6) have been verified. Hypothesis (H1) is clear and the other hypotheses were established in Lemmas 7.1 and 7.2.

Finally we briefly discuss Example 1.2. 
EXAMPLE 1.2. Define $H$ on $L^{2}\left(\mathbf{R}^{n}\right)(n \geq 2)$ formally by

$$
H=H_{0}+a r^{-\beta} \sin b r^{\alpha} g_{1}(x)+a^{\prime} r^{-\beta^{\prime}} \sin b^{\prime} r^{\alpha^{\prime}}\left(1-g_{1}(x)\right)+V+V_{s} \quad(r=|x|),
$$

where $\alpha+\beta>1$ and $2 \alpha+\beta>3 ; \beta^{\prime}+\alpha^{\prime}<1$ and $\alpha^{\prime}<0 ; a, b, a^{\prime}, b^{\prime} \in \mathbf{R}$ and $g_{1} \in C^{\infty}\left(\mathbf{R}^{n}\right)$ satisfies (1.4), $V$ satisfies (LR1) and $V_{s}$ acts multiplicatively and satisfies $(\mathrm{SR})_{1 / 2}$.

It may assist the reader's understanding if we note that the cut-off functions $g_{1}$ and $1-g_{1}$ are only intended to separate the singularities at 0 and at $\infty$ and are not completely necessary. Also note that $\beta<0$ is possible so that completeness of the wave operators means quantum particles must tunnel through the potential walls.

It is worthwhile noting what types of terms $V_{s}$ may incorporate. Since $V_{s}$ acts multiplicatively, it commutes with $e^{U}\left(U \text { as in (LR1)) and so } V_{s} e^{U} \text { satisfies (SR) }\right)_{1 / 2}$ if and only if $V_{s}$ does. Also $V_{s}$ may incorporate local singularities; for example $V_{s}(x)=|x-a|^{-1+\varepsilon}(1+|x|)^{-2 \varepsilon}\left(a \in \mathbf{R}^{n} ; \varepsilon>0\right)$. Another possibility is that $V_{s}(x) \rightarrow 0$ as $|x| \rightarrow \infty$ and $V_{s}$ is some directional derivative $D$ of a function $U_{1}$, satisfying (LR2): $V_{s}=(D) U_{1}-U_{1}(D)$.

These observations are useful because $V_{s}$ will absorb unwanted terms. We define

$$
U(x)=-\frac{2 a}{\alpha^{2} b^{2}} g_{1}(x) r^{-\beta-2 \alpha+2} \sin b r^{\alpha}-\frac{2 a^{\prime}}{\left(\alpha^{\prime}\right)^{2}\left(b^{\prime}\right)^{2}}\left(1-g_{1}(x)\right) r^{-\beta^{\prime}-2 \alpha^{\prime}+2} \sin b^{\prime} r^{\alpha^{\prime}}
$$

Then if we compute $\frac{1}{2} \Delta U+\frac{1}{2}|\nabla U|^{2}$ we get the two oscillatory terms of the potential of $H$ plus terms that satisfy (SR $)_{1 / 2}$ plus one nonoscillatory term from $|\nabla U|^{2}$ satisfying (LR1). Adjusting $V+V_{s}$ appropriately we see that $H$ satisfies the hypotheses of Theorem 1.1.

Appendix. Here we provide proofs of Theorem 2.2, and Lemma 6.3 and 6.4. The proof of Theorem 2.2 is based on the following lemma.

LEMMA A.1. Let $K$ be a compact subset of an open set $U \subseteq \mathbf{R}^{n}$. For any $m \in \mathbf{N}$ and $C^{\prime}>0$, there is $C>0$ so that

$$
\left|\int e^{i \omega h(\xi)} \phi(\xi) d \xi\right| \leq C(1+|\omega|)^{-m} \sup _{0 \leq|\alpha| \leq m} \sup _{\xi \in K}\left|D^{\alpha} \phi(\xi)\right| \quad \text { for all real } \omega,
$$

for all $\phi \in C_{0}^{m}(K)$ and all real $h$ in $C^{m+1}(U)$ such that $|\nabla h(\xi)|>1 / C^{\prime}$ if $\xi \in K$ and $\left|D^{\alpha} h(\xi)\right|<C^{\prime}$ if $\xi \in U, 1 \leq|\alpha| \leq m+1$.

For a proof of this result see [15, Lemma A.1 or 42, p. 37].

PROOF OF THEOREM 2.2. We consider the case $t \geq 0$ only, because the other case follows similarly, or by taking the complex conjugate (by (2.4)).

To show $R_{\sigma}^{-}(f) \phi \equiv f\left(H_{0}\right) \tau_{\eta}^{*} F\left(M_{\sigma}^{-}\right) \tau_{\eta} f\left(H_{0}\right) \phi$ belongs to $S\left(\mathbf{R}^{n}\right)$ whenever $\phi$ does, it suffices to show that $\left(\tau_{\eta}^{*} F\left(M_{\sigma}^{-}\right) \Psi\right)$ belongs to $C^{\infty}\left(\mathbf{R}^{n}\right)$ if $\Psi \in S\left(\mathbf{R}^{2 n}\right)$ (by Lemma 2.1). But this is clear from (2.5).

(a) Set $g=f_{1} f$. To establish (a), we shall prove that

$$
F\left(M_{-\sigma_{1}}^{+}\right) \tau_{\eta} e^{-i t H_{0}} g\left(H_{0}\right) \tau_{\eta}^{*} F\left(M_{\sigma}^{-}\right)
$$


is compact and has suitably bounded operator norm. If $\Psi \in L^{2}\left(\mathbf{R}^{2 n}\right)$, then

$$
\begin{aligned}
& \left(F\left(M_{-\sigma_{1}}^{+}\right) \tau_{\eta} e^{-i t H_{0}} g\left(H_{0}\right) \tau_{\eta}^{*} F\left(M_{\sigma}^{-}\right) \Psi\right)(x, q) \\
& \quad=\chi_{M_{-\sigma}^{+}}(x, q)\left(F\left(M_{\sigma}^{-}\right) \Psi \mid \tau_{\eta} e^{i t H_{0}} \bar{g}\left(H_{0}\right) \eta_{x q}\right) \\
& \quad=\chi_{M_{-\sigma_{1}}^{+}}(x, q) \iint_{M_{\sigma}^{-}}\left(e^{-i t H_{0}} g\left(H_{0}\right) \eta_{y p} \mid \eta_{x q}\right) \Psi(y, p) d y d p
\end{aligned}
$$

where $\chi_{M}$ denotes the indicator function for the set $M$. Thus we have an integral operator with kernel $k(x, q, y, p)$, where

$$
\begin{aligned}
k(x, q, y, p)= & \chi_{M_{-\sigma_{1}}^{+}}(x, q) \chi_{M_{\sigma}^{-}}(y, p) \\
& \cdot \int_{\mathbf{R}^{n}} e^{i(x-y) \cdot \xi-i t|\xi|^{2} / 2} g\left(\frac{1}{2}|\xi|^{2}\right) \hat{\eta}(\xi-q) \hat{\eta}(\xi-p) d \xi .
\end{aligned}
$$

Since $k$ has compact support in $p$ and $q$, it suffices to show that

$$
k(x, q, y, p) \leq C_{m}(1+\omega)^{-m}
$$

for any $m \in \mathbf{N}$, where $\omega=|x|+|y|+t$ for then $\|k\|_{L^{2}\left(\mathbf{R}^{n}\right)} \leq C_{l}(1+t)^{l}$ for any $l \in \mathbf{N}$ and this implies the compactness of $R_{-\sigma_{1}}^{+}\left(f_{1}\right) e^{-i t H} R_{\sigma}^{-}(f)$ and the required bound for its operator norm (by [40, Theorem VI.23, p. 210]. To establish (A.1), we need only apply Lemma A.1 when

$$
h(\xi)=\frac{1}{\omega}\left((x-y) \cdot \xi-\frac{1}{2} t|\xi|^{2}\right) .
$$

The only hypothesis of Lemma A.1 which is not trivially verified is that $|\nabla h(\xi)|$ is bounded below, i.e. for some $c>0$

$$
|x-y-t \xi|>c \omega
$$

whenever $(x, q) \in M_{-\sigma_{1}}^{+},(y, p) \in M_{\sigma}^{-},|\xi-q|<\delta,|\xi-p|<\delta$ and $g\left(\frac{1}{2}|\xi|^{2}\right) \neq 0$. To verify this, one first shows that $(x, \xi) \in M_{-\sigma_{3}}^{+}$and $(y, \xi) \in M_{\sigma_{2}}^{-}$for some $\sigma_{2}, \sigma_{3}$ such that $\sigma_{1}<\sigma_{3}<\sigma_{2}<\sigma$, provided $\delta$ is sufficiently small. (The choice of $\delta$ depends only on $\sigma-\sigma_{2}$ and $\sigma_{3}-\sigma_{1}$, and on the lower bound for the support of g.) This proves that the angle between $x$ and $\xi$ is strictly larger than the angle between $y$ and $\xi$ so that (one easily checks) $|x-y|>c^{\prime}(|x|+|y|)$ for some $c^{\prime}>0$. Replacing $y$ in this argument by $y+t \xi$ and noting $|y+t \xi|>c^{\prime \prime}(|y|+t|\xi|)$ gives (A.2) and establishes (a) of Theorem 2.2.

(b) We derive (b) (when $t>0$ ) from (c) and afterwards prove (c). For any $\phi \in L^{2}\left(\mathbf{R}^{n}\right)$

$$
\begin{aligned}
\left\|R_{\sigma}^{+}(f) e^{-i t H_{0}} \phi\right\| \leq & \left\|R_{\sigma}^{+}(f) e^{-i t H_{0}} F(|x|<b|t|)\right\|\|\phi\| \\
& +\left\|R_{\sigma}^{+}(f)\right\|\|F(|x| \geq b|t|) \phi\|
\end{aligned}
$$

and the first term on the right goes to 0 as $t \rightarrow \infty$ by (c) and the second term clearly goes to 0 . This proves (b).

(c) If we show that, whenever $b>0$ is sufficiently small

$$
\left\|F(|x|<b t) e^{-i t H_{0}} f\left(H_{0}\right) \tau_{\eta}^{*} F\left(M_{\sigma}^{-}\right)\right\| \leq C_{l}(1+t)^{-l}, \quad t>0
$$


for every $l \in \mathbf{N}$ then the result will follow. By (2.5) we have

$$
\begin{aligned}
F(|x| & \left.<b t) e^{-i t H_{0}} f\left(H_{0}\right) \tau_{\eta}^{*} F\left(M_{\sigma}^{-}\right) \Psi\right)(x) \\
& =\chi|x|<b t(x) \int_{\mathbf{R}^{n}} \iint_{M_{\sigma}^{-}} e^{i \xi \cdot(x-y)-(i / 2) t|\xi|^{2}} f\left(\frac{1}{2}|\xi|^{2}\right) \hat{\eta}(\xi-q) \Psi(y, q) d y d q d \xi
\end{aligned}
$$

for $\Psi$ in $L^{1}\left(\mathbf{R}^{2 n}\right) \cap L^{2}\left(\mathbf{R}^{2 n}\right)$. Thus we have an integral operator from $L^{2}\left(\mathbf{R}^{2 n}\right)$ to $L^{2}\left(\mathbf{R}^{n}\right)$ with kernel $K$ where

$$
K(x, y, q)=\chi|x|<b t(x) \chi_{M_{\sigma}^{-}}(y, q) \int_{\mathbf{R}^{n}} e^{i(\xi \mid x-y)-(i / 2) t|\xi|^{2}} f\left(\frac{1}{2}|\xi|^{2}\right) \hat{\eta}(\xi-q) d \xi .
$$

It therefore suffices to prove that, for any $m \in \mathbf{N}$

$$
\|K(x, y, q)\| \leq C_{m}(1+\omega)^{-m}
$$

where $\omega=|y|+t$. (Notice $K(x, y, q)$ has compact support in $q$.)

Thus we need only prove (A.3) for suitable $b>0$ and $\delta>0$, and this is done by applying Lemma A.1 when

$$
h(\xi)=\frac{1}{\omega}\left[\xi \cdot(x-y)-\frac{1}{2} t|\xi|^{2}\right] .
$$

As above, the only hypothesis of Lemma A.1 which is not trivially satisfied is that $|\nabla h(\xi)|$ is bounded below, i.e. for some $c>0$,

$$
|x-y-t \xi|>c \omega
$$

whenever $|x|<b t,(y, p) \in M_{\sigma}^{-},|\xi-p|<\delta$, and $f\left(\frac{1}{2}|\xi|^{2}\right) \neq 0$. To verify this, first observe that $(y, \xi) \in M_{\sigma_{2}}^{-}$for some $\sigma_{2},-1<\sigma_{2}<\sigma$, provided $\delta$ is small enough ( $\delta$ depends on $\sigma-(-1)$ and the lower bound of the support of $f$ ). It follows that $|y+t \xi|>c^{\prime}(|y|+t|\xi|)$ so that

$$
|x-y-t \xi| \geq c^{\prime}(|y|+t|\xi|)-b|t|>c(|y|+t)
$$

for some $c>0$ provided $b$ is small enough ( $b$ too depends on $\sigma+1$ and the lower bound of the support of $f$ ). Thus Lemma A.1 is applicable and (A.3) is established and this completes the proof.

PROOF OF LEMMA 6.3. It suffices to prove equation (6.3) for this will prove that $Q^{ \pm} R_{\sigma}^{ \pm}(f)$ are the limit in operator norm of the compact operators $F(|x| \leq r) Q^{ \pm} f\left(H_{0}\right)$ and hence are compact. We prove the "-" case of $(6.2)$ only; the "+" case is similar. (Or use (5.5) and (2.4).)

For $\phi \in S\left(\mathbf{R}^{n}\right)$ we have, by $(2.5)$

$$
\begin{aligned}
& Q^{-} R_{\sigma}^{-}(f) \phi(x)=\int_{\mathbf{R}^{n}} \iint_{M_{\sigma}^{-}} e^{i \xi \cdot(x-y)} q_{1}(x, \xi)\left\{1-\chi_{\sigma^{\prime}, \sigma}\left(\frac{x \cdot \xi}{|x||\xi|}\right)\right\} \\
& \overline{f\left(\frac{1}{2}|\xi|^{2}\right)} \hat{\eta}(\xi-p)\left(\tau_{\eta} f\left(H_{0}\right) \phi\right)(y, p) d y d p d \xi \\
& =\iint_{M_{\sigma}^{-}} \int_{\mathbf{R}^{n}}\langle x-y\rangle^{-2 N} e^{i \xi \cdot(x-y)}\left(1-\Delta_{\xi}\right)^{N} \\
& \cdot\left[q_{1}(x, \xi)\left\{1-\chi_{\sigma^{\prime}, \sigma}\left(\frac{x \cdot \xi}{|x||\xi|}\right)\right\} \overline{f\left(\frac{1}{2}|\xi|^{2}\right)} \hat{\eta}(\xi-p)\right] \\
& \cdot\left(\tau_{\eta} f\left(H_{0}\right) \phi\right)(y, p) d \xi d y d p
\end{aligned}
$$


where we have written $e^{i \xi \cdot(x-y)}=\langle x-y\rangle^{-2 N}\left(1-\Delta_{\xi}\right)^{N} e^{i \xi \cdot(x-y)}(N \in \mathbf{N})$ and integrated by parts.

Now observe that, for some constant $c>0$

$$
|x-y|>c(|x|+|y|)
$$

provided $(y, q) \in M_{\sigma}^{-},(x, \xi) \notin M_{1 / 3\left(2 \sigma+\sigma^{\prime}\right)}^{-},|\xi-q|<\delta$ and $f\left(\frac{1}{2}|\xi|^{2}\right) \neq 0$ (i.e. on the support of the above integrand) and provided $\delta$ is small enough. The proof of (A.4) is the same as that of (A.2) (when $t=0$ there) in the proof of Theorem 2.2 above.

From the hypotheses we have

$$
\begin{aligned}
& \mid\left(1-\Delta_{\xi}\right)^{N}\left[q_{1}(x, \xi)\right.\left.\left\{1-\chi_{\sigma^{\prime}, \sigma}\left(\frac{x \cdot \xi}{|x||\xi|}\right)\right\} f\left(\frac{1}{2}|\xi|^{2}\right) \hat{\eta}(\xi-p)\right] \mid \\
&<C_{N}\langle x\rangle^{2 N\left(1-\varepsilon_{0}\right)}
\end{aligned}
$$

Combining (A.4) and (A.5) with the earlier expression for $Q^{-} R_{\sigma}^{-}(f) \phi$ we see that

$$
\left|Q^{-} R_{\sigma}^{-}(f) \phi(x)\right| \leq C_{N} \iiint\left(1+|x|^{2}+|y|^{2}\right)^{-N \varepsilon_{0}}\left|\left(\tau_{\eta} f\left(H_{0}\right) \phi\right)(y, p)\right| d y d p d \xi,
$$

where the integral is over $\left\{(y, p, \xi):|\xi-p|<\delta\right.$ and $\xi$ is in the support of $\left.f\left(\frac{1}{2}|\xi|^{2}\right)\right\}$. Since $\tau_{\eta} f\left(H_{0}\right)$ is bounded and $N$ is arbitrary this implies (6.3) (by Schwarz's inequality).

PROOF OF LEMMA 6.4. Make the following decomposition:

$$
\begin{aligned}
F(|x|< & \left.\frac{1}{3} r\right) Q R_{\sigma}^{ \pm}(f) F\left(\left|x^{\prime}\right|>r\right) \\
= & F\left(|x|<\frac{1}{3} r\right) Q \bar{f}\left(H_{0}\right) \tau_{\eta}^{*} F\left(M_{\sigma}^{ \pm}\right) F\left(\left\{(y, p):|y|>\frac{2}{3} r\right\}\right) \tau_{\eta} f\left(H_{0}\right) F\left(\left|x^{\prime}\right|>r\right) \\
& +F\left(|x|<\frac{1}{3} r\right) Q \bar{f}\left(H_{0}\right) \tau_{\eta}^{*} f\left(M_{\sigma}^{ \pm}\right) F\left(\left\{(y, p):|y|<\frac{2}{3} r\right\}\right) \tau_{\eta} f\left(H_{0}\right) F\left(\left|x^{\prime}\right|>r\right) .
\end{aligned}
$$

We shall prove that, for any $l \in \mathbf{N}$

$$
\begin{gathered}
\left\|F\left(|x|<\frac{1}{3} r\right) Q \bar{f}\left(H_{0}\right) \tau_{\eta}^{*} F\left(\left\{(y, q):|y|>\frac{2}{3} r\right\}\right)\right\|<C_{l}(1+r)^{-l}, \\
\left\|F\left(\left\{(y, q):|y|<\frac{2}{3} r\right\}\right) \tau_{\eta} f\left(H_{0}\right) F\left(\left|x^{\prime}\right|>r\right)\right\|<C_{l}(1+r)^{-l}
\end{gathered}
$$

In view of the above decomposition, these inequalities will establish the result provided one also notes that $\left\|F\left(|x|<\frac{1}{3} r\right) Q f\left(H_{0}\right)\right\|<C r^{n / 2}$ (by $[\mathbf{4 0}$, Theorem VI. 23, p. 210]).

The proof of (A.6) is like the proof of Lemma 6.3. For $\Psi \in S\left(\mathbf{R}^{2 n}\right)$ we have, by (2.5)

$$
\begin{aligned}
& \left\|F\left(|x|<\frac{1}{3} r\right) Q \bar{f}\left(H_{0}\right) \tau_{\eta}^{*} F\left(\left\{(y, p):|y|>\frac{2}{3} r\right\}\right) \Psi\right\|^{2} \\
& =\int_{|x|<r / 3} \mid \int_{\mathbf{R}^{n}} \int_{\mathbf{R}^{n}} \int_{|y|>2 r / 3} e^{i \xi \cdot(x-y)} q_{2}(x, \xi) \bar{f}\left(\frac{1}{2}|\xi|^{2}\right) \\
& \left.\cdot \hat{\eta}(\xi-p) \Psi(y, p) d y d p d \xi\right|^{2} d x \\
& =\int_{|x|<r / 3} \mid \int_{\mathbf{R}^{n}} \int_{\mathbf{R}^{n}} \int_{|y|>2 r / 3}\langle x-y\rangle^{-2 N} e^{i \xi \cdot(x-y)}\left(1-\Delta_{\xi}\right)^{N} \\
& \left.\cdot\left\{q_{2}(x, \xi) \bar{f}\left(\frac{1}{2}|\xi|^{2}\right) \hat{\eta}(\xi-p)\right\} \Psi(y, p) d y d p d \xi\right|^{2} d x
\end{aligned}
$$


where we have written $e^{i \xi \cdot(x-y)}=\langle x-y\rangle^{-2 N}\left(1-\Delta_{\xi}\right)^{N} e^{i \xi(x-y)}$ and integrated by parts. Since $|x|<\frac{1}{3} r$ and $y>\frac{2}{3} r,\langle x-y\rangle>C\langle y\rangle>C^{\prime}(1+r)>C^{\prime \prime}\langle x\rangle$, for positive constants $C, C^{\prime}, C^{\prime \prime}$. By hypothesis

$$
\left|\left(1-\Delta_{\xi}\right)^{N}\left\{q_{2}(x, \xi) f\left(\frac{1}{2}|\xi|^{2}\right) \hat{\eta}(\xi-p)\right\}\right|<C_{N}\langle x\rangle^{2 N\left(1-\varepsilon_{1}\right)}
$$

so that

$$
\begin{aligned}
& \left\|F\left(|x|<\frac{1}{3} r\right) Q \bar{f}\left(H_{0}\right) \tau_{\eta}^{*} F\left(\left\{(y, p):|y|<\frac{2}{3} r\right\}\right) \Psi\right\|^{2} \\
& <C(1+r)^{-2 l} \int \mid \iint\langle x-y\rangle^{-2 N \varepsilon_{1}+2 l+(n+1) / 2} \\
& \left.\cdot\langle y\rangle^{-(n+1) / 2}|\Psi(y, p)| d y d p d \xi\right|^{2} d x
\end{aligned}
$$

where the integral is over $\left\{(x, \xi, p, y):|x|<\frac{1}{3} r, \xi\right.$ in the support of $f\left(\frac{1}{2}|\xi|^{2}\right)$, $\left.|p-\xi|<\delta, y \in \mathbf{R}^{n}\right\}$. Applying Schwarz' inequality establishes (A.6). (Take $2 N \varepsilon_{1}>2 l+\frac{1}{2}(n+1)$.)

Inequality (A.7) follows similarly. We have

$$
\begin{aligned}
& F\left(\left\{(y, p):|y|<\frac{2}{3} r\right\}\right) \tau_{\eta} f\left(H_{0}\right) F\left(\left|x^{\prime}\right|>r\right) \phi \\
& \quad=F\left(\left\{(y, p):|y|<\frac{2}{3} r\right\}\right)\left(\phi F\left(\left|x^{\prime}\right|>r\right) \bar{f}\left(H_{0}\right) \eta_{y p}\right)
\end{aligned}
$$

which is an integral operator from $L^{2}\left(\mathbf{R}^{n}\right)$ to $L^{2}\left(\mathbf{R}^{2 n}\right)$ with kernel

$$
\begin{aligned}
k\left(y, p, x^{\prime}\right) & =\chi_{|y|<2 r / 3}(y) \chi_{\left|x^{\prime}\right|>r}\left(x^{\prime}\right)\left(f\left(H_{0}\right) \eta_{y p}\right)\left(x^{\prime}\right) \\
& =\chi_{|y|<2 r / 3}(y) \chi_{\left|x^{\prime}\right|>r}\left(x^{\prime}\right) \int_{\mathbf{R}^{n}} e^{i \xi \cdot\left(x^{\prime}-y\right)} f\left(\frac{1}{2}|\xi|^{2}\right) \hat{\eta}(\xi-p) d \xi
\end{aligned}
$$

by the Fourier inversion formula. Thus (A.7) will follow if we show that the $L^{2}\left(\mathbf{R}^{3 n}\right)$ norm of $k$ is bounded by $C_{l}(1+r)^{l}$. As before we write

$$
e^{i \xi \cdot\left(x^{\prime}-y\right)}=\left\langle x^{\prime}-y\right\rangle^{-2 N}\left(1-\Delta_{\xi}\right)^{N} e^{i \xi \cdot\left(x^{\prime}-y\right)}
$$

and integrate by parts:

$$
\begin{aligned}
k\left(y, p, x^{\prime}\right)= & \chi_{|y|<2 r / 3}(y) \chi_{\left|x^{\prime}\right|>r}\left(x^{\prime}\right)\left\langle x^{\prime}-y\right\rangle^{-2 N} \\
& \cdot \int_{\mathbf{R}^{n}} e^{i \xi \cdot\left(x^{\prime}-y\right)}\left(1-\Delta_{\xi}\right)^{N}\left\{f\left(\frac{1}{2}|\xi|^{2}\right) \hat{\eta}(\xi-p)\right\} d \xi
\end{aligned}
$$

Since $\left\langle y-x^{\prime}\right\rangle>C\left\langle x^{\prime}\right\rangle>C^{\prime}(1+r)>C^{\prime \prime}\langle y\rangle$ and $k\left(y, p, x^{\prime}\right)$ is completely supported in $p,\|k\|_{L^{2}}<C_{l}(1+r)^{-l}$ as required. (Choose $N$ so that $2 N>l+n+1$.)

ACKNOWLEDGEMENTS. It is a pleasure to thank Professor H. Kitada for discussing his work and making his manuscripts available prior to publication and also to thank Professor Lon Rosen for his interest in the two-Hilbert space approach. I also thank Professor Rosen, Dr. Joel Feldman and the University of British Columbia for their hospitality during the preparation of this paper.

\section{REFERENCES}

1. S. Agmon, Some new results in spectral and scattering theory of differential operators in $\mathbf{R}^{n}$, Séminaire Goulaouic-Schwarz, 1978-79, Centre de Mathématiques-Polytechnique, Palaiseau.

2. A. P. Calderón and R. Vaillancourt, A class of bounded pseudo-differential operators, Proc. Nat. Acad. Sci. U.S.A. 69 (1972), 1185-1187. 
3. M. Combescure, Spectral and scattering theory for a class of strongly oscillating potentials, Comm. Math. Phys. 73 (1980), 43-62.

4. A. Devinatz and P. Rejto, A limiting absorption principle for Schrödinger operators with oscillating potentials, part I, J. Differential Equations 49 (1983), 29-84.

5. __ A limiting absorption principle for Schrödinger operators with oscillating potentials, part II, J. Differential Equations 49 (1983), 85-104.

6. J. D. Dollard, Asymptotic convergence and the Coulomb interactions, J. Math. Phys. 5 (1964), 729-738.

7. _ Quantum-mechanical scattering theory for short-range and Coulomb interactions, Rocky Mountain J. Math. 1 (1971), 5-88.

8. J. D. Dollard and C. N. Friedman, Existence of Moller wave operators for $V(r)=$ $\lambda \sin \left(\mu r^{\alpha} / r^{\beta}\right)$, Ann. Physics 111 (1978), 251-266.

9. V. Enss, Asymptotic completeness for quantum mechanical potential scattering, I. Short range potentials, Comm. Math. Phys. 61 (1978), 285-291.

10. __ Asymptotic completeness for quantum mechanical potential scattering, II. Singular and long range potentials, Ann. Physics 119 (1979), 117-132.

11. _ Geometric methods in spectral and scattering theory of Schrödinger operators, Rigorous Atomic and Molecular Physics (G. Velo and A. S. Wightman, Eds.), Plenum Press, New York, 1981.

12. __ Asymptotic observables on scattering states, Comm. Math. Phys. 89 (1983), 245268.

13. __ Propagation properties of quantum scattering states, J. Funct. Anal. 52 (1983), 219-251.

14. J. Ginibre, La méthode 'dépendant du temps' dans le problème de la complétude asymptotique, preprint Univ. Paris-Sud LPTHE 80/10, 1980.

15. L. Hormander, The existence of wave operators in scattering theory, Math. Z. 146 (1976), 69-91.

16. T. Ikebe and H. Isozaki, Completeness of modified wave operators for long-range potentials, Publ. Res. Inst. Math. Sci. Kyoto Univ. 15 (1979), 679-718.

17. _ A stationary approach to the existence and completeness of long-range wave operators, Integral Equations and Operator Theory 5 (1982), 18-49.

18. H. Isozaki, On generalized Fourier transforms associated with long-range perturbations, J. Reine Angew. Math. 337 (1982), 18-67.

19. __ Eikonal equation and spectral representations for long-range Schrödinger Hamiltonians, J. Math. Kyoto Univ. 20 (1980), 243-261.

20. H. Isozaki and H. Kitada, Modified wave operators with time-independent modifiers, J. Fac. Sci. Univ. Tokyo Sect. IA 32 (1985), 77-104.

21. __ Scattering matrices for two-body Schrödinger operators, preprint.

22. __ Asymptotic behaviour of the scattering amplitude at high energies, Differential Equations, ed. by I. W. Knowles and R. T. Lewis, Elsevier, North-Holland, 1984, pp. 327334.

23. T. Kato, Existence and equivalence of two types of long-range modified wave operators, J. Fac. Sci. Univ. Tokyo Sect. IA 25 (1978), 133-147.

24. __ Perturbation theory for linear operators, 2nd ed., Springer-Verlag, Berlin, 1976.

25. H. Kitada, Scattering theory for Schrödinger operators with long-range potentials. I, J. Math. Soc. Japan 29 (1977), 665-691.

26. __ Scattering theory for Schrödinger operators with long-range potentials. II, J. Math. Soc. Japan 30 (1978), 603-632.

27. __ Scattering theory for Schrödinger equations with time-independent potentials of long-range type, J. Fac. Sci. Univ. Tokyo Sect. IA 29 (1982), 353-369.

28. $\_$A calculus of Fourier integral operators and the global fundamental solution for a Schrödinger equation, Osaka J. Math. 19 (1982), 863-900.

29. __ Time decay of the high energy part of the solution for a Schrödinger equation, J. Fac. Sci. Univ. Tokyo Sect. IA 31 (1984), 109-146.

30. H. Kitada and K. Yajima, A scattering theory for time-independent long-range potentials, Duke Math. J. 49 (1982), 341-376. 
31. __ Remarks on our paper, 'A scattering theory for time-independent long-range potentials', Duke Math. J. 50 (1983), 1005-1016.

32. E. Mourre, Link between the geometrical and the spectral transformation approachs in scattering theory, Comm. Math. Phys. 68 (1979), 91-94.

33. PL. Muthuramalingam and K. Sinha, Asymptotic completeness in long-range scattering, II, Ann. Scuola Norm. Sup. Pisa 18 (1985), 57-88.

34. D. B. Pearson, Scattering theory for a class of oscillating potentials, Helv. Phys. Acta 52 (1979), 541-554.

35. P. A. Perry, Mellin transformations and scattering theory. I, short-range potentials, Duke Math. J. 47 (1980), 187-193.

36. __ Scattering theory by the Enss method, Mathematical Reports, No. 1, Harwood, New York, 1983.

37. _ Propagation of states in dilation analytic potentials and asymptotic completeness, Comm. Math. Phys. 81 (1981), 243-259.

38. E. Prugovecki, On time-independent scattering for long-range interactions, Nuovo Cimento 4B (1971), 105-127.

39. E. Prugovecki and J. Zorbas, Modified Lippman-Schwinger equations for two-body scattering theory with long-range interactions, J. Math. Phys. 14 (1973), 1398-1409.

40. M. Reed and B. Simon, Methods of modern mathematical physics, I. Functional analysis, Academic Press, New York, 1975.

41. _ Methods of modern mathematical physics, II. Fourier analysis, self adjointness, Academic Press, New York, 1975.

42. _ Methods of modern mathematical physics, III. Scattering theory, Academic Press, New York, 1979.

43. _ Methods of modern mathematical physics, IV. Analysis of operators, Academic Press, New York, 1978.

44. B. Simon, Phase space analysis of simple scattering systems: extensions of some work of Enss, Duke Math. J. 46 (1979), 119-168.

45. Y. Saito, Spectral representations for Schrödinger operators with long-range potentials, Lecture Notes in Math., vol. 727, Springer-Verlag, Berlin, 1979.

46. K. B. Sinha and PL. Muthuramalingam, Asymptotic evolution of certain observables and completeness in Coulomb scattering. I, J. Funct. Anal. 55 (1984), 323-343.

47. D. White, Schrödinger operators with rapidly oscillating central potentials, Trans. Amer. Math. Soc. 275 (1983), 641-677.

48. E. B. Davies, On Enss' approach to scattering theory, Duke Math. J. 47 (1980), 171-185.

Department of MATHEMATiCs, University of British Columbia, VANCOUVER, British COLUMBia V6T 1W5, CANADA

Current address: Department of Mathematics, University of Toledo, Toledo, Ohio 43606 